COMMUNICATIONS IN

ANALYSIS AND GEOMETRY

Volume 12, Number 3, 511-551, 2004

\title{
Quasiconvex Foliations and Asymptotically Flat Metrics of Non-negative Scalar Curvature
}

\author{
Brian Smith and Gilbert Weinstein
}

We prove that a broad subset of the space of asymptotically flat Riemannian metrics of nonnegative scalar curvature on $\mathbb{R}^{3}$ is connected using a new method for prescribing scalar curvature that generalizes a method developed by Bartnik for quasi-spherical metrics.

\section{Introduction.}

Asymptotically flat Riemannian 3-dimensional metrics of non-negative scalar curvature arise naturally in general relativity when one considers the Einstein vacuum constraint equations in the maximal gauge. Indeed, the normalnormal and normal-tangential components of the Einstein vacuum equations in that gauge read:

$$
\begin{gathered}
R=|k|^{2}, \\
\operatorname{div} k=0, \quad \operatorname{tr} k=0,
\end{gathered}
$$

where $R$ is the scalar curvature of the Riemannian metric $g$ induced on a maximal time-slice, and $k$ is the second fundamental form of that slice in the ambient Lorentzian 4-manifold. It is clear from (1) that $R \geq 0$. The remaining Einstein vacuum equations can be seen as governing the evolution of the data consisting of the first and second fundamental form $g$ and $k$. Since this evolution traces a continuous path in the space of initial data with the appropriate topology, it is natural to ask: what are the topological properties of this space of data? In particular, a question of considerable importance is whether this space is connected. It is possible to show using the conformal method that two sets of initial data $(g, k),\left(g^{\prime}, k^{\prime}\right)$ are in the same pathconnected component of the space of initial data if and only the metrics $g$ and $g^{\prime}$ are in the same path-connected component of the space of metrics of non-negative scalar curvature [17].

A topological 2-sphere in a Riemannian manifold is said to be quasiconvex if its Gauss and mean curvatures are positive. A foliation is quasiconvex 
if its leaves are quasiconvex spheres. We say that a Riemannian metric $g$ on $\mathbb{R}^{3}$ is quasiconvex if for some point $x \in \mathbb{R}^{3}, \mathbb{R}^{3} \backslash\{x\}$ admits a quasiconvex foliation. In this paper we develop a new method for constructing quasiconvex asymptotically flat Riemannian metrics of nonnegative scalar curvature. As our main application we prove that the set of quasiconvex asymptotically flat Riemannian metrics of nonnegative scalar curvature on $\mathbb{R}^{3}$ is connected. Our proof uses a nonstandard method for prescribing scalar curvature that involves solving a parabolic reaction-diffusion equation on $\mathbb{S}^{2}$ for an undetermined metric component, where a radial coordinate on $\mathbb{R}^{3}$ takes the place of time, and the coordinate spheres of $\mathbb{R}^{3}$ have been identified with $\mathbb{S}^{2}$. This method for the prescription of scalar curvature is a generalization of a method developed by Bartnik for quasi-spherical metrics [2]. Our result was announced in [18].

Let $r$ be the standard Euclidean distance from the origin in $\mathbb{R}^{3}$. We will use the weighted Hölder spaces $C_{-\tau}^{k, \alpha}$ defined by:

$$
\begin{aligned}
\|f\|_{C_{-\tau}^{k}} & =\sum_{i=0}^{k}\left\|\sigma^{\tau+i} D^{i} f\right\|_{C^{0}} \\
{[f]_{\alpha,-\tau} } & =\sup _{x, y} \sigma(x, y)^{\tau+\alpha} \frac{|f(x)-f(y)|}{|x-y|^{\alpha}} \\
\|f\|_{C_{-\tau}^{k, \alpha}} & =\|f\|_{C_{-\tau}^{k}}+\left[D^{k} f\right]_{C_{-\tau-k}^{\alpha}} \\
C_{-\tau}^{k, \alpha} & =\left\{f:\|f\|_{C_{-\tau}^{k, \alpha}}<\infty\right\} .
\end{aligned}
$$

Here $k$ is a positive integer, $\alpha \in(0,1), \tau \in(0,1], \sigma(x)=\sqrt{1+|x|^{2}}$, $\sigma(x, y)=\min \{\sigma(x), \sigma(y)\}$, and $D^{i} f$ is the $i$-th order derivative of $f$. For a tensorfield $T$ on $\mathbb{R}^{3}$ we define $\|T\|_{C_{-\tau}^{k, \alpha}}$ to be the sum of the $C_{-\tau}^{k, \alpha}$ norms of the coordinate components. We note that although these norms are coordinate dependent, the topology they induce is invariant under diffeomorphisms of bounded distortion. We say that a diffeomorphism $\Phi: \mathbb{R}^{3} \rightarrow \mathbb{R}^{3}$ is of bounded distortion in $C_{-\tau}^{k, \alpha}$ if there is a rigid rotation $\Theta$ of $\mathbb{R}^{3}$ such that $\Theta \circ \Phi-I \in C_{-\tau}^{k, \alpha}$. It is straightforward to check that if the transition function between two coordinate systems is a diffeomorphism of bounded distortion in $C_{-\tau}^{k, \alpha}$ then the two norms defined with these coordinate systems are equivalent, and hence the topologies induced by these norms are equal. 
In addition, we will also use the space $C_{*}^{2}$ defined by:

$$
\begin{aligned}
\|f\|_{C_{*}^{2}} & =\sum_{i=1}^{2}\left\|\sigma^{i} D^{i} f\right\|_{C^{0}} \\
C_{*}^{2} & =\left\{f:\|f\|_{C_{*}^{2}}<\infty\right\} .
\end{aligned}
$$

Let $S_{r}$ denote the coordinate spheres, and let $\kappa, \chi$, and $H$ be, respectively, the Gauss, extrinsic, and mean curvatures of $S_{r}$ considered as functions on $\left(\mathbb{R}^{3} \backslash\{O\}, g\right)$. Let $\alpha \in(0,1)$, and let $\delta$ be the standard metric of $\mathbb{R}^{3}$. Define $\mathcal{M}_{\alpha}$ to be the space of Riemannian metrics on $\mathbb{R}^{3}$ satisfying:

(i) $g-\delta \in C_{-1}^{2, \alpha}$;

(ii) $g(0)=\delta$;

(iii) $R(g) \geq 0$;

(iv) $H, \kappa>0$.

Condition (iv) requires that the foliation spheres $\left\{S_{r}\right\}$ are quasiconvex, i.e., have positive mean and Gauss curvatures. Henceforth the subscript $\alpha$ will be dropped so that we have $\mathcal{M}=\mathcal{M}_{\alpha}$. Let $\mathcal{M}$ be equipped with the topology induced by $C_{-1}^{2, \alpha}$. Let $\mathcal{G}$ denote the group of diffeomorphisms of $\mathbb{R}^{3}$ with bounded distortion in $C_{-1}^{2, \alpha}$. We can now state our main theorem.

Main Theorem. The quotient $\mathcal{M} / \mathcal{G}$ is path connected in the quotient topology.

As mentioned earlier, this theorem is proved using a parabolic equation for prescribing scalar curvature. This equation relates the scalar curvature $R(g)$ of a metric $g$ to the component of $g$ normal to the foliation $\left\{S_{r}\right\}$. Write $g=u^{2} d r \otimes d r+r^{2} \gamma$, with the 2-tensor $\gamma$ vanishing on the unit normal $N$ to the foliation, and define $\bar{N}=r u N, \bar{\chi}=r^{-1} u \chi, \bar{H}=r u H$, and $\bar{\kappa}=r^{2} \kappa$. Then, it can be shown that $\bar{\chi}, \bar{H}$, and $\bar{\kappa}$ can be computed in terms of $\gamma$ and $\bar{N}$ only. Using $\not>$ and $\phi_{\gamma}$ to denote the covariant derivative and the Laplacian with respect to $\gamma$ on $S_{r}$, we have

$$
\bar{H} \not_{\bar{N}} u=u^{2} \not_{\gamma} u+\bar{A} u-\bar{B} u^{3},
$$

where

$$
\begin{gathered}
\bar{A}=\not_{\bar{N}} \bar{H}-\bar{H}+\frac{1}{2}|\bar{\chi}|_{\gamma}^{2}+\frac{1}{2} \bar{H}^{2}, \\
\bar{B}=r^{2}\left(\kappa-\frac{1}{2} R\right)=e^{-2 v}(1-\not \downarrow v v)-\frac{1}{2} r^{2} R ;
\end{gathered}
$$


see [18].

To study this equation as a reaction-diffusion equation we make an identification of $\mathbb{R}^{3} \backslash\{0\}$ with $\mathbb{R}^{+} \times \mathbb{S}^{2}$ using a family of diffeomorphisms $\pi_{r}: S_{r} \rightarrow \mathbb{S}^{2}$. This family is chosen so that applying the Poincaré uniformization separately on each $S_{r}$ we can write $\gamma=e^{2 v} \bar{\gamma}$ where $\left(\pi_{r}\right)_{*} \bar{\gamma}$ is a fixed round metric on $\mathbb{S}^{2}$ (independent of $r$ ). Equation (3) then becomes

$$
r \partial_{r} u-\beta \cdot \not \downarrow u=\Gamma u^{2} \not \Delta u+A u-B u^{3},
$$

where $\Gamma=e^{-2 v} / \bar{H}, A=\bar{A} / \bar{H}, B=\bar{B} / \bar{H}, \not \Delta=\not_{\bar{\gamma}}$, and $\not$ is the covariant derivative with respect to $\bar{\gamma}$, and $\beta=r \partial_{r}-\bar{N}$. We then rewrite the metric $g$ as:

$$
g=u^{2} d r^{2}+e^{2 v} \bar{\gamma}_{A B}\left(\beta^{A} d r+r d \theta^{A}\right)\left(\beta^{B} d r+r d \theta^{B}\right),
$$

where $\beta$ is the shift vector defined above and $\left(\theta^{1}, \theta^{2}\right)$ are local coordinates pulled back from $\mathbb{S}^{2}$ under the mappings $\pi_{r}$. It will be shown in Section 2 that this is possible for any $g \in \mathcal{M}$ after a small perturbation. Given a triple $(v, \beta, R)$, our aim will be to show that Equation (4) can under appropriate conditions be solved for the normal component $u$ to produce, by substituting into (5), a metric $g$ with the prescribed scalar curvature $R$. The condition $\bar{H}>0$ in (iv) guarantees that (4) is parabolic, while the condition $\bar{\kappa}>0$ is used to prevent blowup.

To deform a given $g \in \mathcal{M}$ we define the deformation explicitly on a coordinate ball $B_{r_{0}}$, while on its complement $\mathbb{R}^{3} \backslash B_{r_{0}}$ we make a deformation of the data $(v, \beta, R)$ with $\bar{H}, \bar{\kappa}>0, R \geq 0$. We use these as sources in equation (4), and the resulting family of equations is then solved for a deformation of the normal component $u$, where for initial data we take the deformation of $u$ already given on $S_{r_{0}}$ by the deformation on $B_{r_{0}}$. This device is used to avoid dealing with the somewhat delicate analytical subtleties associated with giving initial conditions for (4) at $r=0$ where the equation is singular.

The outline of the paper is as follows: In Section 2 we first show that given a smooth family of metrics $\gamma_{\lambda}$ on $\mathbb{S}^{2}$ depending differentiably on a parameter $\lambda$ there exists a smooth family of conformal factors $\phi_{\lambda}$, differentiable in $\lambda$, such that $\bar{\gamma}_{\lambda}=\left(\phi_{\lambda}\right)^{-1} \gamma$ is round for every $\lambda$. Next, we use this result to show that any sufficiently smooth metric $g \in \mathcal{M}$ that is conformally flat near the origin and at infinity can be rewritten as in (5). In Section 3 we show that any $g \in \mathcal{M}$ can be deformed in $\mathcal{M}$ to a metric that is $C_{-1}^{k}$ for all integers $k$ and that is conformally flat at the origin and at infinity; this is needed in order to apply the results of Section 2 and also so that we will not lose any differentiability when we transfer to the parabolic Hölder spaces used 
in Section 4. Section 3 uses the conformal method and also makes use of a number of elliptic results for weighted Hölder spaces, which are stated there and proved in the appendix. In Section 4 we collect the analytical results we need concerning Equation (4). Finally, in Section 5 we make a sequence of metric deformations as outlined in the previous paragraph to construct a path of metrics starting at an arbitrary metric $g_{0} \in \mathcal{M}$ and ending at a flat metric.

\section{Uniformization of Foliation Spheres.}

Our main goal in this section is to write a given metric in the form (5). For this purpose we must, for each $r \in \mathbb{R}^{+}$, find a uniformization of the induced metric on the foliation sphere $S_{r}$. The resulting 1-parameter family of conformal factors, which will be indexed by $r$, must be not only differentiable on $\mathbb{S}^{2}$, but also differentiable in $r$. In addition, we must have appropriate behavior as $r$ approaches 0 and $\infty$. As will be seen in Section 3 we can assume that $g$ is conformally flat at infinity and at the origin. Thus, given a family of metrics $\gamma$ on $\mathbb{S}^{2}$ that depend smoothly on a parameter $r \in[a, d]$ and for which a uniformizing conformal factor is already given for $r \in[a, b] \cup[c, d] \subset[a, d]$, we are to construct a smooth family of uniformizing conformal factors on $[a, d]$ that agrees with the one given on a neighborhood of the endpoints $a$ and $d$. This is essentially the content of Theorem 5 . To accomplish this we use a modification of the uniformization of $\mathbb{S}^{2}$ in [7] and then show that the resulting family of conformal factors has the appropriate dependence on $r$.

Here, as in [7], the uniformization is accomplished by first choosing $P \in \mathbb{S}^{2}$ and constructing a function $w$, singular at $P$, such that $\gamma_{0}=e^{2 w} \gamma$ is a flat metric on $\mathbb{S}^{2} \backslash\{P\} \approx \mathbb{R}^{2}$. The standard stereographic projection then gives another conformal factor $e^{2 \tilde{u}}$ so that $\bar{\gamma}=e^{2(w+\tilde{u})} \gamma$ has Gauss curvature identically equal to 1 . Regularity theory then shows that $w+\tilde{u}$ is smooth across $P$, and the desired conformal factor has been constructed. The primary difference between our uniformization and that of [7] is in the construction of the function $w$. In [7], this function is given on a neighborhood of $P$ by $v-2 \log (\tilde{\lambda})$, where $\tilde{\lambda}$ is the geodesic distance from $P$, and $v$ verifies $\boldsymbol{X}_{\gamma} v=\kappa(\gamma)+2 \boldsymbol{\not}_{\gamma} \log (\tilde{\lambda})$ on this neighborhood. We modify this by replacing $\tilde{\lambda}$ with $\lambda=\sqrt{x^{2}+y^{2}}$, which is defined in terms of local isothermal coordinates $(x, y)$. This has the advantage that it is more readily apparent that $w$ and then $\tilde{u}$ are also differentiable in the parameter on $\mathbb{S}^{2} \backslash\{P\}$. In Theorem 4 we show that the family of conformal factors is appropriately differentiable in the parameter at $P$ as well so that we will have $w+\tilde{u} \in C^{\infty}\left(I \times \mathbb{S}^{2}\right)$. 
Let the parameter interval be denoted by $I$. It follows from the next propositions that $\tilde{u}, w \in C^{\infty}\left(I \times \mathbb{S}^{2} \backslash\{P\}\right)$. The first two of these involve the differentiable dependence upon a parameter of the solutions of the elliptic partial differential equation

$$
\not_{\gamma} v=f
$$

on subsets of $\mathbb{S}^{2}$, where $\gamma$ is a family of metrics on $\mathbb{S}^{2}$ and $f$ is a family of functions, both of which depend smoothly on a parameter $r$. The third proposition involves the differentiable dependence on the parameter of the exponential map and its inverse.

Proposition 1. Let $\Omega \subset \mathbb{S}^{2}, \bar{\Omega} \neq \mathbb{S}^{2}$ with $C^{\infty}$ boundary $\partial \Omega$. Let $\gamma \in$ $C^{\infty}(I \times \bar{\Omega})$ be a family of metrics on $\mathbb{S}^{2}$, and let $f \in C^{\infty}(I \times \bar{\Omega})$ be a family of functions. Then there exists a unique family of solutions $v \in C^{\infty}(I \times \Omega)$ of Equation (6) with the boundary data $\left.v\right|_{\partial \Omega}=0$.

To prove this proposition we regard $\Omega$ as a domain of $\mathbb{R}^{2}$. The result follows from Theorem 6.8 and Exercise 6.2 of [10] together with the fact that if a family of isomorphisms $L$ is smooth in the parameter then $L^{-1}$ will be also.

Proposition 2. Let $\gamma, f \in C^{\infty}\left(I \times \mathbb{S}^{2}\right)$ satisfy $\int_{\mathbb{S}^{2}} f d A_{\gamma}=0$ for every value of the parameter, and let $P \in \mathbb{S}^{2}$. Then there exists a unique family of solutions $v \in C^{\infty}\left(I \times \mathbb{S}^{2}\right)$ of Equation (6) satisfying $v(P)=0$.

Proof. Since $\int f d A_{\gamma}=0$, standard elliptic theory on $\mathbb{S}^{2}$ shows that there exists a unique family of solutions $v$ of Equation (6) on $\mathbb{S}^{2}$ satisfying $v(P)=$ 0 . To show that $v$ is smooth in $r$ we consider the family of operators $L$ : $C^{k+2, \alpha}\left(\mathbb{S}^{2}\right) \rightarrow C^{k, \alpha}\left(\mathbb{S}^{2}\right)$ defined by

$$
L(w)=\not_{\gamma} w+w(P), \quad P \in \mathbb{S}^{2} .
$$

To see that $L$ is an isomorphism note that if $w, h$ satisfy

$$
L(w)=h,
$$

then we have $w(P)=\bar{h}$, where $\bar{h}$ is the average of $h$. From this observation we have that $\phi_{\gamma} w=h-\bar{h}$ from which, using standard elliptic theory, we derive a bound of the form

$$
\|w\|_{C^{k+2, \alpha}} \leq C\|h\|_{C^{k, \alpha}} .
$$

Furthermore, we can solve $\not \mathcal{X} w^{\prime}=h-\bar{h}$ and add a constant to get a solution $w=w^{\prime}-w^{\prime}(P)+\bar{h}$ of (7). Thus, a bounded family of inverses $L^{-1}$ exists, 
which is seen to be smooth in $r$. Since we have $v=L^{-1} f$ it follows that $v \in C^{\infty}\left(I, C^{k+2, \alpha}\left(\mathbb{S}^{2}\right)\right)$. This holds for all integers $k$, and thus we get $v \in$ $C^{\infty}\left(I \times \mathbb{S}^{2}\right)$

Given a family of metrics $\gamma$ on $\mathbb{S}^{2}$ and a point $P \in \mathbb{S}^{2}$, we will use $\exp _{\gamma, P}$ to denote the corresponding family of exponential maps at the point $P$. The next proposition follows from dependence on parameters in ordinary differential equations and the inverse function theorem.

Proposition 3. Let $P \in \mathbb{S}^{2}$ and let $W$ be a neighborhood of $P$. Suppose that $\gamma \in C^{\infty}(I \times W)$. Let $V \subset T_{P} M$ be a compact set containing 0 such that $\exp _{\gamma, P}(V) \subset W$ and $\exp _{\gamma, P}: V \rightarrow W$ is injective for every value of the parameter. Let $U \subset \mathbb{S}^{2}$ satisfy $U \subset \exp _{\gamma, P}(V)$ for every value of the parameter. Then $\exp _{\gamma, P} \in C^{\infty}(I \times V, W)$ and $\exp _{\gamma, P}^{-1} \in C^{\infty}(I \times U, V)$.

With the help of Propositions 1 and 3 we can now construct, on a neighborhood $B$ of $P$, a family of isothermal coordinates $(x, y) \in C^{\infty}(I \times B)$ for the family $\gamma$. Let $\Omega$ be a $C^{\infty}$ neighborhood of $P$, and let $\kappa(\gamma)$ be the family of Gauss curvatures of $\gamma$. With $f=\kappa(\gamma)$ and $\left.v^{\prime}\right|_{\partial \Omega}=0$, let $v^{\prime} \in C^{\infty}(I \times \Omega)$ be the family of solutions of (6) given by proposition 1 . On $\Omega$ we have that $\tilde{\gamma}_{0}=e^{2 v^{\prime}} \gamma$ is a family of flat metrics. Let $B \subset \Omega$ be such that $\exp _{\tilde{\gamma}_{0}, P}$ is injective on $\exp _{\tilde{\gamma}_{0}, P}^{-1}(B)$. Proposition 3 can be used to obtain a family of Cartesian normal coordinates $(x, y) \in C^{\infty}(I \times B)$ associated with $\tilde{\gamma}$ and with origin at $P$

With $\lambda=\sqrt{x^{2}+y^{2}}$, the family $\log \lambda$ verifies $\not_{\gamma} \log \lambda=0$ on $B \backslash\{P\}$ and is clearly $C^{\infty}(I \times B \backslash\{P\})$. Let $\varphi$ be a positive $C^{\infty}$ cutoff function identically 1 on a neighborhood of $P$ and supported on $B$. We have

$$
\int_{\mathbb{S}^{2}} \phi_{\gamma}(\varphi \log \lambda) d V_{\gamma}=-2 \pi
$$

for every value of the parameter. This equation is obtained by noting that for $\varepsilon$ small enough

$$
\int_{\mathbb{S}^{2}} \boldsymbol{\phi}_{\gamma}(\varphi \log \lambda) d V_{\gamma}=\int_{\mathbb{R}^{2} \backslash B_{\varepsilon}(0)}\left(\partial_{x}^{2}+\partial_{y}^{2}\right)\left(\varphi \log \left(\sqrt{x^{2}+y^{2}}\right)\right) d x d y
$$

followed by an application of the divergence theorem to the right hand side.

Now from (8) and the Gauss Bonnet Theorem we see that $\int(\kappa(\gamma)+$ $2 \not \lambda \varphi \log \lambda)=0$. It follows from Proposition 2 that there is a unique family of solutions $v \in C^{\infty}\left(I \times \mathbb{S}^{2}\right)$ of

$$
\boldsymbol{\phi}_{\gamma} v=\kappa(\gamma)+2 \boldsymbol{\not}_{\gamma}(\varphi \log \lambda), v(P)=0
$$


Defining $w=v-2 \varphi \log \lambda, \gamma_{0}=e^{2 w} \gamma$ is flat. Define $\rho$ to be the geodesic distance from point $O$ with respect to $\gamma_{0}$. By proposition 3 it follows that $\rho \in C^{\infty}\left(I \times \mathbb{S}^{2} \backslash\{P\}\right)$, and hence $\tilde{u}=-\log \left(1+\frac{\rho^{2}}{4}\right) \in C^{\infty}\left(I \times \mathbb{S}^{2} \backslash\{P\}\right)$. Defining $u=w+\tilde{u}$, it can be checked that the Gauss curvature of the metric $e^{2 u} \gamma$ is identically equal to 1 on $\mathbb{S}^{2} \backslash P$.

As in [7], if we can verify an $L^{\infty}$-bound on $u$ for each value of the parameter $r$ it follows from $L^{p}$-estimates that $u(\tau) \in W^{2, p}\left(\mathbb{S}^{2}\right)$ for every $p>0$ [7]. Sobolev embedding theorems along with standard bootstrap arguments will then give $u \in C^{\infty}\left(\mathbb{S}^{2}\right)$ for each $r$. To derive the $L^{\infty}$-bound note that on a neighborhood of $P$ we can write

$$
\gamma_{0}=e^{2\left(w-v^{\prime}\right)} \tilde{\gamma}_{0}=\lambda^{-4} e^{2\left(v-v^{\prime}\right)}\left(d \lambda^{2}+\lambda^{2} d \theta^{2}\right) .
$$

Thus we get a bound of the form $c^{-1} \lambda^{-1}<\rho<c \lambda^{-1}$ and the $L^{\infty}$ bound on $u=\tilde{u}+w$ follows. We conclude that $\bar{\gamma}=e^{2 u} \gamma$ is a round $C^{\infty}$ metric for each $r$. Define $\phi=e^{-2 u}$. We refer to the factorization $\gamma=\phi \bar{\gamma}$ as a $(P, O)$-uniformization. We note that $O$ and $P$ are antipodal points for $\mathbb{S}^{2}$ with respect the metric $\bar{\gamma}$ for every $r$. We now need to show that in fact $\phi \in C^{\infty}\left(I \times \mathbb{S}^{2}\right)$.

Theorem 4. Let $\gamma \in C^{\infty}\left(I \times \mathbb{S}^{2}\right)$. Then there is a $(P, O)$ uniformization $\gamma=\phi \bar{\gamma}$ with $\phi \in C^{\infty}\left(I \times \mathbb{S}^{2}\right)$.

Proof. Take $\gamma=\phi \bar{\gamma}$ to be the $(P, O)$-uniformization constructed above. Then from the observations of the previous two paragraphs we have $\phi, \bar{\gamma} \in C^{\infty}(I \times$ $\left.\mathbb{S}^{2} \backslash\{P\}\right)$. Let $\gamma=\phi^{\prime} \bar{\gamma}^{\prime}$ be an $(O, P)$-uniformization. Then $\phi^{\prime}, \bar{\gamma}^{\prime} \in C^{\infty}(I \times$ $\left.\mathbb{S}^{2} \backslash\{O\}\right)$. Let $z$ be stereographic coordinates for $\bar{\gamma}$ projected from $P$ and let $z^{\prime}$ be stereographic coordinates for $\bar{\gamma}^{\prime}$ projected from $O$. Since $z, z^{\prime}$ can be constructed in terms of normal coordinates of $\bar{\gamma}$ and $\bar{\gamma}^{\prime}$, respectively, it follows from Proposition 3 that $z \in C^{\infty}\left(I \times \mathbb{S}^{2} \backslash\{P\}, \mathbb{C}\right)$ and $z^{\prime} \in C^{\infty}(I \times$ $\left.\mathbb{S}^{2} \backslash\{O\}, \mathbb{C}\right)$. Since $\bar{\gamma}=\left(\phi^{\prime} / \phi\right) \bar{\gamma}^{\prime}$, the metrics $\bar{\gamma}, \bar{\gamma}^{\prime}$ are related by a conformal diffeomorphism for every value of the parameter, and thus $z=\left(a z^{\prime}+b\right) /\left(c z^{\prime}+\right.$ $d)$. Since $z$ is projected from $P$ and $z^{\prime}$ is projected from $O$ it follows that $a=d=0$, and we have $z=\alpha / z^{\prime}$ where $\alpha=b / c$ is a function from $I$ into $\mathbb{C}$. For some point $N \neq O, P$ it is clear that $z(N), z^{\prime}(N) \in C^{\infty}(I, \mathbb{C})$, from which it follows that $\alpha \in C^{\infty}(I, \mathbb{C})$. Defining $\tilde{z}=2 / z$ it is immediate that $\tilde{z} \in C^{\infty}\left(I \times \mathbb{S}^{2} \backslash\{O\}, \mathbb{C}\right)$, and since we can write

$$
\bar{\gamma}=\left(1+\frac{|\tilde{z}|^{2}}{4}\right)^{-2} d|\tilde{z}|^{2}
$$


we have also $\bar{\gamma} \in C^{\infty}\left(I \times \mathbb{S}^{2} \backslash\{O\}\right)$. We already know that $\bar{\gamma} \in C^{\infty}(I \times$ $\left.\mathbb{S}^{2} \backslash\{P\}\right)$, and thus $\bar{\gamma} \in C^{\infty}\left(I \times \mathbb{S}^{2}\right)$. The theorem follows since, for instance, $\phi=\operatorname{det}(\gamma) / \operatorname{det}(\bar{\gamma})$.

Theorem 5. Let $\gamma \in C^{\infty}\left(I \times \mathbb{S}^{2}\right)$ with $I=[0,2]$. Suppose that on the subinterval $[1,2]$ a $(P, O)$ uniformization $\gamma=\phi^{\prime} \bar{\gamma}^{\prime}$ is given with $\phi^{\prime} \in$ $C^{\infty}\left([1,2] \times \mathbb{S}^{2}\right)$. Then there is a $(P, O)$-uniformization of class $C^{\infty}\left(I \times \mathbb{S}^{2}\right)$ that agrees with $\gamma=\phi^{\prime} \bar{\gamma}^{\prime}$ on $\left[\frac{3}{2}, 2\right]$.

Proof. Let $\gamma=\tilde{\phi} \tilde{\bar{\gamma}}$ be the $(P, O)$-uniformization on the interval $I=[0,2]$ given by Theorem 4 . Let $z$ be stereographic coordinates projected from the point $P$. Then on $[1,2]$ we have that $\bar{\gamma}^{\prime}=\psi_{\alpha} \tilde{\bar{\gamma}}$, where

$$
\psi_{\alpha}=\left\{\frac{1+|z|^{2} / 4}{\alpha+\alpha^{-1}|z|^{2} / 4}\right\}^{2}
$$

and $\alpha \in C^{\infty}([1,2], \mathbb{R})$. Let $0 \leq \lambda \leq 1$ be a $C^{\infty}$ cutoff function supported on $[1,2]$ and satisfying $\lambda \equiv 1$ on $[3 / 2,2]$. Define

$$
\tilde{\alpha}=\lambda \alpha+(1-\lambda) \text {. }
$$

Then on $[1,2]$ we have $\gamma=\left(\tilde{\phi} \psi_{\tilde{\alpha}}^{-1}\right)\left(\psi_{\tilde{\alpha}} \tilde{\bar{\gamma}}\right)$. Thus $\phi \equiv \tilde{\phi} \psi_{\tilde{\alpha}}^{-1}$ gives the required uniformization.

Theorem 6. Suppose $g-\delta \in C_{-1}^{\infty}$ satisfies $g=e^{2 \tilde{v}} \delta$ on a neighborhood of the origin and outside a compact set. Then $g$ can be written in the form

$$
g=u^{2} d r^{2}+e^{2 v} \bar{\gamma}_{A B}\left(\beta^{A} d r+r d \theta^{A}\right)\left(\beta^{B} d r+r d \theta^{B}\right),
$$

where $\bar{\gamma}_{A B}$ is a fixed round metric on the foliation spheres $S_{r}$ expressed in terms of local coordinates $\theta^{1}, \theta^{2}$ on $\mathbb{S}^{2}$. Furthermore, defining $\beta=\beta^{A} \partial / \partial_{\theta^{A}}$, it can be arranged that $u-1, v, \sigma^{-1} \beta \in C_{-1}^{\infty}$.

Proof. Let $\gamma$ be defined by

$$
\gamma=r^{-2}\left(g-u^{2} d r \otimes d r\right),
$$

where $u^{-2}=g(\nabla r, \nabla r)$. Let $N$ denote the unit outward normal to the foliation $\left\{S_{r}\right\}$. Since $u d r(N)=1$ it follows that $\gamma(N, \cdot)=0$. When there is no possible ambiguity we also use $\gamma$ to denote $\left.\gamma\right|_{S_{r}}$. By hypothesis $u=e^{\tilde{v}}$ outside a compact set and on a neighborhood of the origin; whence $u-1 \in C_{-1}^{\infty}$. 
Let $F=(r, \omega): \mathbb{R}^{3} \backslash\{0\} \rightarrow \mathbb{R}^{+} \times \mathbb{S}^{2}$ be the standard spherical coordinates on $\mathbb{R}^{3}$. View $\omega$ instead as a family of mappings, which for every $r$ maps $S_{r}$ onto $\mathbb{S}^{2}$. We conclude using Theorem 4 that we can write $\omega_{*} \gamma=e^{2 v} \bar{\gamma}^{\prime}$ for $v \in C^{\infty}\left(\mathbb{R}^{+} \times \mathbb{S}^{2}\right)$. We abuse notation slightly and use $v$ also to denote the function $\omega^{*} v$ defined on $\mathbb{R}^{3} \backslash\{0\}$. Using Theorem 5 we can arrange for $v$ to agree with $\tilde{v}$ outside of a compact set and in a neighborhood of the origin; hence $v \in C_{-1}^{\infty}$.

Let $l: \mathbb{S}^{2} \rightarrow \mathbb{S}^{2}$ be a smooth family of diffeomorphisms such that $l_{*} \bar{\gamma}^{\prime}$ is fixed in the parameter $r$ and such that $l$ is the identity for $r$ large and small. Define $\omega^{\prime}=l \circ \omega$. Let $X$ be the tangent vectorfield to the family of curves $\left(\omega^{\prime}\right)^{-1}(p), p \in \mathbb{S}^{2}$ that satisfies $d r(X)=1$. Defining $\bar{\gamma}=\left(\omega^{\prime}\right)^{*} \bar{\gamma}^{\prime}$, we have $\bar{\gamma}(X, \cdot)=0, \mathcal{L}_{X} \bar{\gamma}=0$, and $\left.\gamma\right|_{S_{r}}=\left.e^{2 v} \bar{\gamma}\right|_{S_{r}}$.

Let $\theta^{1}, \theta^{2}$ be local coordinates on $\mathbb{S}^{2}$. We abuse notation and use $\theta^{1}, \theta^{2}$ to denote also the functions $\theta^{1} \circ \omega^{\prime}, \theta^{2} \circ \omega^{\prime}$, which give local angular coordinates on $\mathbb{R}^{3}$. Let $\bar{N} \equiv \operatorname{ruN}$ and define $\beta^{1}, \beta^{2}$ by $\beta^{A}=-\bar{N}\left(\theta^{A}\right)$. Let the one forms $d \phi^{1}, d \phi^{2}$ be defined by $d \phi^{A}=\beta^{A} d r+r d \theta^{A}$. Since $d \phi^{1}, d \phi^{2}$ are linearly independent and vanish on $N$, we can write $r^{2} \gamma=\gamma_{A B} d \phi^{A} d \phi^{B}$. The form (9) is obtained by using local coordinates $\left(r, \theta^{1}, \theta^{2}\right)$ and substituting $\bar{\gamma}=$ $\bar{\gamma}_{A B} d \theta^{A} d \theta^{B}$ in $\left.\gamma\right|_{S_{r}}=\left.e^{2 v} \bar{\gamma}\right|_{S_{r}}$ to conclude $\gamma_{A B}=e^{2 v} \bar{\gamma}_{A B}$. Denote $\partial_{A}=$ $\partial / \partial \theta^{A}$, and define the shift vectorfield $\beta=\beta^{A} \partial_{A}$. Applying the identity operator $\partial_{r} \otimes d r+\partial_{A} \otimes d \theta^{A}$ to $\bar{N}$, and using $\bar{N}(r)=r, X=\partial_{r}$, we obtain $\beta=r \partial_{r}-\bar{N}$. Noting that $\beta$ vanishes outside of a compact set and on a neighborhood of the origin, it follows immediately that $\sigma^{-1} \beta \in C_{-1}^{\infty}$.

This gives a representation of the metric $g$ in terms of the functions $u, v$ and the vectorfield $\beta$. Note that setting $u=1, v=0, \beta=0$ in (9) gives a flat metric that in general is not equal to $\delta$. Define $F^{\prime}=\left(r, \omega^{\prime}\right)$, where $\omega^{\prime}$ is as in the proof of the theorem above. Then the metric $g^{\prime}=\left(F^{-1} \circ F^{\prime}\right)_{*} g$ can be written in the form (9), where the coordinates $\theta^{1}, \theta^{2}$ are now standard angular coordinates on $\mathbb{R}^{3}$. Setting $u=1, v=0, \beta=0$ in the transformed metric $g^{\prime}$ would give the standard flat background metric $\delta$. For simplicity, we will often replace $g$ by $g^{\prime}$.

In view of $\mathcal{L}_{\partial_{r}} \bar{\gamma}=0$, the extrinsic curvature $\chi$ of the foliation can be calculated rather simply. Define $\Pi$ to be the orthogonal projection from $T_{p} \mathbb{R}^{3}$ to $T_{p} S_{r}$. Let $\gamma$ be as in the proof of the theorem and let $\hat{\beta}$ denote the one form $\hat{\beta}_{B} d \theta^{B}$, where $\hat{\beta}_{B}=\gamma_{A B} \beta^{A}$. Since $\mathcal{L}_{\bar{N}} d r$ vanishes on $T S_{r}$, we have:

$$
\chi=\frac{\Pi}{2} \mathcal{L}_{N}\left(r^{2} \gamma\right)=\frac{\Pi}{2 r u} \mathcal{L}_{\bar{N}}\left(r^{2} e^{2 v} \bar{\gamma}-\operatorname{sym}(\xi \otimes d r)\right)=\frac{\Pi}{2 r u} \mathcal{L}_{\bar{N}}\left(r^{2} e^{2 v} \bar{\gamma}\right)
$$


where $\xi=2 r \hat{\beta}+\hat{\beta}(\beta) d r$. Writing $\bar{N}=r \partial_{r}-\beta$ and using $\mathcal{L}_{\partial_{r}} \bar{\gamma}=0$, we get

$$
\chi=\frac{r}{u}\left(\left(1+r \partial_{r} v\right) \gamma-\frac{1}{2} \mathcal{L}_{\beta} \gamma\right) .
$$

Taking the trace with respect to $g$ gives the mean curvature,

$$
H=\frac{1}{r u}\left(2\left(1+r \partial_{r} v\right)-\operatorname{div}_{\gamma} \beta\right),
$$

or equivalently

$$
H=\frac{1}{r u}\left(2\left(1+r \partial_{r} v\right)-e^{-2 v} \operatorname{div} \bar{\gamma}(\hat{\beta})\right) .
$$

In the metric deformations made later in the paper we always take the covariant form $\hat{\beta}$, which for simplicity will be denoted also as $\beta$ so that the last equation is written

$$
H=\frac{1}{r u}\left(2\left(1+r \partial_{r} v\right)-e^{-2 v} \operatorname{div}_{\bar{\gamma}} \beta\right) .
$$

The Gauss curvature of the foliation is given by

$$
\kappa=r^{-2} e^{-2 v}(1-\not \Delta v)
$$

Define $\bar{H}=r u H$ and $\bar{\kappa}=r^{2} \kappa$.

The asymptotic behavior of $u$ as $r \rightarrow \infty$ is important in establishing asymptotic flatness. Thus, following Bartnik [1], we define the auxiliary function

$$
m=r\left(1-u^{-2}\right)
$$

to aid in the study of this behavior.

\section{Preliminary Deformations.}

As remarked in the introduction, the deformation of a given metric in $\mathcal{M}$ is largely accomplished by solving (4) along a parameter, where the results of the last section are used to assume that the starting metric is of the form (9). However, the results of that section require that the metric $g$ satisfies $g-\delta \in C_{-1}^{\infty}$ and in addition that $g$ is conformally flat near $\infty$ and in a neighborhood of the origin. In this section it is shown that an initial deformation can be made so that the resulting metric has these properties. In 3.2 we show that any $g_{0} \in \mathcal{M}$ can be continuously deformed in $\mathcal{M}$ to a 
metric $g_{1} \in \mathcal{M} \cap\left(\delta+C_{-1}^{\infty}\right)$ that is conformal to $\delta$ near infinity. In 3.3 it is shown that any such $g_{1}$ can be deformed in $\mathcal{M}$ to a metric $g_{2} \in \mathcal{M} \cap\left(\delta+C_{-1}^{\infty}\right)$ that, in addition to being conformal to $\delta$ near infinity, is also conformal to $\delta$ in a neighborhood of the origin. Most of the results of this section are obtained using standard elliptic theory involving the weighted Hölder spaces $C_{-\tau}^{k, \alpha}$; the basic elliptic results needed are collected in 3.1 .

In both 3.2 and 3.3 the deformations are accomplished by explicitly defining a deformation $\tilde{g} \in C\left([0,1], \delta+C_{-1}^{2, \alpha}\right)$ so that $\tilde{g}(0)=g_{0}$ and $\tilde{g}$ would be in $\mathcal{M}$ but for the sign of the scalar curvature. We then find $\psi \in C\left([0,1], C_{-1}^{2, \alpha}\right)$ such that the path $(1+\psi)^{4} g$ has nonnegative scalar curvature. In order to preserve quasi-convexity, the following proposition is used:

Proposition 7. Let $g \in C_{-1}^{2}$ be such that the coordinate spheres are quasiconvex. There exists a $\delta>0$ such that if

$$
\left\|g-g^{\prime}\right\|_{C_{*}^{2}} \leq \delta
$$

then the coordinate spheres are also quasi-convex with respect to $g^{\prime}$.

Proof. This is proved using the following scale invariance of $\bar{H}, \bar{\kappa},\|\cdot\|_{C_{*}^{2}}$ :

$$
\begin{aligned}
\left.\bar{H}\left(g_{r}\right)\right|_{S_{1}} & =\left.\bar{H}(g)\right|_{S_{r}} \\
\left.\bar{\kappa}\left(g_{r}\right)\right|_{S_{1}} & =\left.\bar{\kappa}(g)\right|_{S_{r}} \\
\left\|g_{r}\right\|_{C^{2}, S_{1}} & =\|g\|_{C_{*}^{2}, S_{r}},
\end{aligned}
$$

where $\left(g_{r}\right)_{i j}=g_{i j}(r x)$. In view of (17)-(18) we can choose $\varepsilon>0$ small enough that $\left.\bar{H}\left(g_{r}\right)\right|_{S_{1}},\left.\bar{\kappa}\left(g_{r}\right)\right|_{S_{1}}>\varepsilon$. Considering $\left.\bar{H}(h)\right|_{S_{1}}=F(p, Q)$ and $\left.\bar{\kappa}(h)\right|_{S_{1}}=G(p, Q)$ as functions of $p \in S_{1}$ and $Q=\left(h, \partial h, \partial^{2} h\right) \in A$, where $A=\left\{Q=\left(h, \partial h, \partial^{2} h\right) \in \mathbb{R}^{60}: \operatorname{det} h>0\right\}$, then $F$ and $G$ are continuous on $S_{1} \times A$. Pick $r>0$, so that $\left\|g-g^{\prime}\right\|_{C_{*}^{2}}<r \operatorname{implies} \operatorname{det} g^{\prime} \geq \mu=\frac{1}{2} \inf \operatorname{det} g$, and note that $\left\|g^{\prime}\right\|_{C_{*}^{2}} \leq\|g\|_{C_{-1}^{2}}+r=C$ for any such $g^{\prime}$. Let $K=B_{C} \cap$ $\{\operatorname{det} h \geq \mu\} \subset \mathbb{R}^{60}$; then $K$ is compact, and hence $F$ and $G$ are uniformly continuous on $S_{1} \times K$. Thus, there is $\delta>0$ such that $\left|F(p, Q)-F\left(p, Q^{\prime}\right)\right|<\varepsilon$ and $\left|G(p, Q)-G\left(p, Q^{\prime}\right)\right|<\varepsilon$ whenever $\left|Q-Q^{\prime}\right|<\delta$. If $\left\|g-g^{\prime}\right\|_{C_{*}^{2}}<\delta$ then $\left\|g_{r}-g_{r}^{\prime}\right\|_{C^{2}, S_{1}}<\delta ;$ hence

$$
\begin{array}{r}
\left|\bar{\kappa}\left(g_{r}\right)-\bar{\kappa}\left(g_{r}^{\prime}\right)\right|_{S_{1}}<\varepsilon \\
\left|\bar{H}\left(g_{r}\right)-\bar{H}\left(g_{r}^{\prime}\right)\right|_{S_{1}}<\varepsilon .
\end{array}
$$

From this we conclude $\left.\bar{H}\left(g_{r}^{\prime}\right)\right|_{S_{1}},\left.\bar{\kappa}\left(g_{r}^{\prime}\right)\right|_{S_{1}}>0$. The identities (17)-(18) then show that $\bar{H}\left(g^{\prime}\right), \bar{\kappa}\left(g^{\prime}\right)>0$. 


\subsection{Elliptic Theory.}

We will need some results about elliptic operators in the Hölder spaces $C_{-\beta}^{k, \alpha}\left(\mathbb{R}^{3}\right)$, which are collected here. However, in order not to stop the main line of the paper, we present the proofs in an appendix. Throughout this subsection $k$ is an integer greater than or equal to 2. The first theorem is an extension of a theorem of Choquet-Bruhat and Chaljub-Simon, which can be found in [9]; see also [14].

Theorem 8. Let $\tau>0$, and let $g \in \delta+C_{-\tau}^{k-1, \alpha}$ be a metric on $\mathbb{R}^{3}$.

(a) Let $u \in C_{-\beta}^{0}$ and $\Delta_{g} u \in C_{-\beta-2}^{k-2, \alpha}$. Then $u \in C_{-\beta}^{k, \alpha}$ and

$$
\|u\|_{C_{-\beta}^{k, \alpha}} \leq C\left(\left\|\Delta_{g} u\right\|_{C_{-\beta-2}^{k-2, \alpha}}+\|u\|_{C_{-\beta}^{0}}\right) .
$$

(b) Let $0<\beta<1, \nu>2$, and let $h \in C_{-\nu}^{k-2, \alpha}$. If the operator $\Delta_{g}-h$ : $C_{-\beta}^{k, \alpha} \rightarrow C_{-\beta-2}^{k-2, \alpha}$ is injective then it is an isomorphism.

When $\beta=1$, we need to add an $L^{1}$-condition on $\Delta u$ to recover the isomorphism result. We incorporate this condition in new Banach spaces. For $k \geq 0$ let $D_{-3}^{k, \alpha}=C_{-3}^{k, \alpha} \cap L^{1}$ be equipped with the norm

$$
\|f\|_{D_{-3}^{k, \alpha}}=\|f\|_{C_{-3}^{k, \alpha}}+\|f\|_{L^{1}}
$$

and for $k \geq 2$ let $E_{-1}^{k, \alpha}=\left\{u \in C_{-1}^{k, \alpha}:\|\Delta u\|_{L^{1}}<\infty\right\}$ be equipped with the norm

$$
\|u\|_{E_{-1}^{k, \alpha}}=\|u\|_{C_{-1}^{k, \alpha}}+\|\Delta u\|_{L^{1}}
$$

where $\Delta$ denotes here the Laplacian with respect to the flat metric $\delta$. It is easy to check that $D_{-3}^{k, \alpha}$ and $E_{-1}^{k, \alpha}$ are Banach spaces.

Theorem 9. Let $\tau>0$, let $g \in \delta+C_{-\tau}^{k-1, \alpha}$ be a metric on $\mathbb{R}^{3}$, and let $h \in C_{-\nu}^{k-2, \alpha}, \nu>2$. If the operator

$$
\Delta_{g}-h: E_{-1}^{k, \alpha} \rightarrow D_{-3}^{k-2, \alpha}
$$

is injective then it is an isomorphism.

Let $G(x, y)$ be the fundamental solution of $\Delta_{g} u=0$; see [16] for the existence of $G$. Then the following representation holds for any $u \in C_{-\beta}^{2, \alpha}$ :

$$
u(x)=-\int_{\mathbb{R}^{3}} \Delta_{g} u(y) G(x, y) d \mu_{g}(y) .
$$


In [16], the following bounds on $G(x, y)$ are proved:

$$
\frac{1}{C|x-y|} \leq G(x, y) \leq \frac{C}{|x-y|}
$$

We will also need weighted higher derivative estimates on $G(x, y)$.

Lemma 10. Let $G(x, y)$ be the fundamental solution of $\Delta_{g} u=0$. Then the following inequalities hold:

$$
\begin{aligned}
|\partial G(x, y)| & \leq \frac{C}{|x-y|^{2}} \\
\left|\partial^{2} G(x, y)\right| & \leq \frac{C}{|x-y|^{3}} .
\end{aligned}
$$

\subsection{The Smoothing Deformation.}

A metric $g$ on $\mathbb{R}^{3}$ is referred to as harmonically flat in the case that, in addition to being asymptotically flat, $R(g)$ is compactly supported and there exists a compact set $B$ such that $g$ is conformal to $\delta$ on $\mathbb{R}^{3} \backslash B .{ }^{1}$ Given any metric $g_{0} \in \mathcal{M}$, we will now construct a continuous deformation of $g_{0}$ to a harmonically flat metric $g_{1} \in C_{-1}^{\infty} \cap \mathcal{M}$. We first find, for any $\mu>0$, a path $\tilde{g}_{t}$ satisfying:

(i) $\tilde{g}_{0}=g_{0}$;

(ii) $\tilde{g}_{1} \in C_{-1}^{\infty}$, and $\tilde{g}_{1}=\delta$ outside a compact set;

(iii) $\tilde{g}_{t}(0)=\delta$;

(iv) $\tilde{g}_{t} \in C^{0}\left(I, C_{-1}^{2, \alpha}\right)$ and $R\left(\tilde{g}_{t}\right) \in C^{0}\left(I, L^{1}\right)$;

(v) $\left\|\tilde{g}_{t}-g_{0}\right\|_{C_{-1 / 2}^{2, \alpha}}<\mu$ and $\left\|R\left(\tilde{g}_{t}\right)-R\left(g_{0}\right)\right\|_{C_{-5 / 2}^{\alpha}}<\mu$.

Once $\tilde{g}_{t}$ is found, the metrics $g_{t}$ can be constructed by the conformal method. Indeed, if $\mu>0$ is chosen small enough then by (v) the operator

$$
\Delta_{\tilde{g}_{t}}-\frac{1}{8} R\left(\tilde{g}_{t}\right): C_{-1 / 2}^{k, \alpha} \rightarrow C_{-5 / 2}^{k-2, \alpha}
$$

\footnotetext{
${ }^{1}$ Previously, these metrics have been referred to as harmonically flat at infinity (see [3]), but we shorten this phrase here for convenenience.
} 
is an isomorphism since it is norm-close to $\Delta_{g_{0}}-R\left(g_{0}\right)$, which is an isomorphism due to $R\left(g_{0}\right) \geq 0$ and part (b) of Theorem 8 . Choose a nonnegative function of compact support $\tilde{R} \in C^{\infty}$ so that

$$
\left\|R\left(g_{0}\right)-\tilde{R}\right\|_{C_{-5 / 2}^{\alpha}}<\mu \text {. }
$$

The family of operators $L_{t}=\Delta_{\tilde{g}_{t}}-\frac{1}{8} R\left(\tilde{g}_{t}\right): E_{-1}^{2, \alpha} \rightarrow D_{-3}^{0, \alpha}$ is continuous in $t$, and by Theorem 9 also isomorphisms. Hence the family of inverses $L_{t}^{-1}: E_{-1}^{2, \alpha} \rightarrow D_{-3}^{0, \alpha}$ is also continuous in $t$. Thus, we obtain a family of solutions $\phi_{t} \in C^{0}\left(I, C_{-1}^{2, \alpha}\right)$ of the equation

$$
\left(\Delta_{\tilde{g}_{t}}-\frac{1}{8} R\left(\tilde{g}_{t}\right)\right) \phi_{t}=\frac{1}{8} R\left(\tilde{g}_{t}\right)-\frac{1}{8} \tilde{R},
$$

which by part (b) of Theorem 8 satisfies

$$
\left\|\phi_{t}\right\|_{C_{-1 / 2}^{2, \alpha}} \leq 2 C \mu
$$

Define $g_{t}$ by:

$$
g_{t}=\left(1+\phi_{t}\right)^{4} \tilde{g}_{t}
$$

It follows immediately that $R\left(g_{t}\right)=\left(1+\phi_{t}\right)^{5} \tilde{R} \geq 0$ and $g_{t} \in C^{0}\left(I, C_{-1}^{2, \alpha}\right)$. Condition (v) and (24) imply $\left\|g_{t}-g_{0}\right\|_{C_{*}^{2}} \leq C \mu$; thus if $\mu$ is small enough it follows from Proposition 7 that the coordinate spheres are also quasi-convex with respect to $g_{t}$. Condition (ii) and Theorem 9 show that $g_{1} \in C_{-1}^{\infty}$.

It remains to construct a deformation $\tilde{g}_{t}$ satisfying (i)-(v). This is done in 2 steps.

Step 1. In this step, we deform the metric $g_{0}$ to a metric that is equal to $\delta$ outside a large ball $B_{2 \rho}$. Let $0 \leq \eta \leq 1$ be a cutoff function that is identically 1 in $B_{1}$ and vanishes outside $B_{2}$, and put $\eta_{\rho}(x)=\eta(x / \rho)$. Define

$$
\tilde{g}_{t}=g_{0}+2 t\left(1-\eta_{\rho}\right)\left(\delta-g_{0}\right)
$$

for $0 \leq t \leq 1 / 2$. Clearly, if $\rho$ is large enough then (v) holds. Define the operator $T$ acting on a metric $h$ by:

$$
T(h)=\partial_{i} \partial_{j} h_{i j}-\partial_{i} \partial_{i} h_{j j}
$$

It is easily checked that if $h \in C_{-1}^{2}$ and $T(h) \in L^{1}$ then $R(h) \in L^{1}$. Since $\eta_{\rho}\left(g_{0}-\delta\right)+\delta=\delta$ outside $B_{2 \rho}$, it follows that $T\left(\eta_{\rho}\left(g_{0}-\delta\right)+\delta\right) \in L^{1}$. Thus by the linearity of $T$,

$$
T\left(\tilde{g}_{t}\right)=(1-2 t) T\left(g_{0}\right)+2 t T\left(\eta_{\rho}\left(g_{0}-\delta\right)+\delta\right) \in L^{1},
$$


and it follows that $R\left(\tilde{g}_{t}\right) \in C^{0}\left([0,1 / 2], L^{1}\right)$.

Step 2. By using a standard smoothing operator we find a metric $h \in C_{0}^{\infty}$ such that $\left\|h-\tilde{g}_{1 / 2}\right\|_{C_{-1}^{2, \alpha}}<\mu$. Note that $|h(0)-\delta|<\mu$. For $1 / 2 \leq t \leq 1$ define

$$
\tilde{g}_{t}=2(1-t) \tilde{g}_{1 / 2}+(2 t-1)(h-\eta(h(0)-\delta)) .
$$

Since

$$
\tilde{g}_{t}-\tilde{g}_{1 / 2}=(2 t-1)\left(h-\tilde{g}_{1 / 2}-\eta(h(0)-\delta)\right)
$$

it follows that $\left\|\tilde{g}_{t}-\tilde{g}_{1 / 2}\right\|_{C_{-1}^{2, \alpha}}<C \mu$, which implies (v).

We have shown:

Proposition 11. Let $g_{0} \in \mathcal{M}$, then there is a continuous path $g_{t} \in \mathcal{M}$ from $g_{0}$ to a harmonically flat metric $g_{1} \in \mathcal{M} \cap C_{-1}^{\infty}$.

\subsection{Local Conformal Flattening.}

Given a harmonically flat metric $g_{1} \in\left\{\delta+\mathcal{M} \cap C_{-1}^{\infty}\right\}$, we now construct a path $g_{t}$ from $g_{1}$ to a metric $g_{2}$ that in addition is conformal to $\delta$ also in a neighborhood of the origin. For each $t, g_{t}$ will remain close enough to $g_{1}$ in $C_{*}^{2}$ for Proposition 7 to guarantee the quasi-convexity condition for $g_{t}$. In order to construct $g_{t}$ we assume that the standard coordinates $\left(x^{1}, x^{2}, x^{3}\right)$ of $\mathbb{R}^{3}$ are normal coordinates for $g_{1}$. At the end of this subsection we will show that there is no loss of generality in making this assumption.

Pick $0<\epsilon<1$. With $\eta_{\varepsilon}$ as in 3.2 and $t \in[1,2]$, we define

$$
\tilde{g}_{t}=g_{1}+(t-1) \eta_{\varepsilon}\left(\delta-g_{1}\right) \text {. }
$$

It is clear that $\tilde{g}_{t}=g_{1}$ on $\mathbb{R}^{3} \backslash B_{2 \varepsilon}$ and $\tilde{g}_{2}=\delta$ on $B_{\varepsilon}$. In addition, since $\left(x^{1}, x^{2}, x^{3}\right)$ are normal coordinates it follows that for $\epsilon>0$ small

$$
\left|\tilde{g}_{i j}-\delta_{i j}\right| \leq C \varepsilon^{2}, \quad\left|\partial_{k} \tilde{g}_{i j}\right| \leq C \varepsilon, \quad\left|\partial_{l} \partial_{k} \tilde{g}_{i j}\right| \leq C
$$

for some constant $C$ that is independent of $t$ and $\varepsilon$. It then follows that there is an $a$, also independent of $\varepsilon$ and $t$, such that

$$
\left|R^{-}\left(\tilde{g}_{t}\right)\right| \leq a(t-1)
$$

Define $\rho_{t}=2 a(t-1) \eta_{2 \varepsilon}$, and note that

$$
\left\|\rho_{t}\right\|_{C^{\alpha}} \leq C \varepsilon^{-\alpha}
$$


In view of Theorem 9 there exists $\phi_{t} \in C^{0}\left([1,2], C_{-1}^{2, \alpha}\right)$ satisfying

$$
\Delta_{g_{t}} \phi_{t}=-\frac{1}{8} \rho_{t}
$$

Note that $\phi_{t} \in C_{-1}^{\infty}$ for all $t$. We now estimate $\phi_{t}$ using the representation (21):

$$
\phi_{t}(x)=\frac{1}{8} \int_{\mathbb{R}^{3}} \rho_{t}(y) G(x, y) d \mu_{g}(y),
$$

and the estimate (22). For $x \in B_{6 \varepsilon}$ we have

$$
\left|\phi_{t}(x)\right| \leq C \int_{B_{4 \varepsilon}} \frac{d y}{|x-y|} \leq C \int_{B_{10 \varepsilon}} \frac{d y}{|y|}=C \varepsilon^{2},
$$

and for $x \in \mathbb{R}^{3} \backslash B_{6 \varepsilon}$

$$
\left|\phi_{t}(x)\right| \leq C \int_{B_{4 \varepsilon}} \frac{d y}{|x-y|} \leq \frac{C}{|x|} \int_{B_{4 \varepsilon}} \frac{d y}{1-|y| /|x|} \leq C \frac{\varepsilon^{3}}{|x|} .
$$

The Inequalities (29) and (30) now imply

$$
\left(-\Delta_{\tilde{g}_{t}}+\frac{1}{8} R\left(\tilde{g}_{t}\right)\right)\left(1+\phi_{t}\right) \geq \frac{1}{8}\left|R^{-}\left(\tilde{g}_{t}\right)\right|\left(1-C \varepsilon^{2}\right) .
$$

Thus, defining

$$
g_{t}=\left(1+\phi_{t}\right)^{4} \tilde{g}_{t}
$$

it follows that $R\left(g_{t}\right) \geq 0$ as long as $\epsilon<1 / \sqrt{C}$.

We now show that $\left\|g_{t}-g_{1}\right\|_{C_{*}^{2}}$ can be made arbitrarily small by choosing $\epsilon>0$ small enough. It will thus follow from Proposition 7 that the quasiconvexity condition holds for $g_{t}$. Note that

$$
\left\|g_{t}-g_{1}\right\|_{C_{*}^{2}} \leq\left\|\tilde{g}_{t}\left(\left(1+\phi_{t}\right)^{4}-1\right)\right\|_{C_{*}^{2}}+\left\|\tilde{g}_{t}-g_{1}\right\|_{C_{*}^{2}}
$$

and by (25) the second term on the right-hand side is small provided $\epsilon>0$ is small. Thus, it remains to bound $\left\|\phi_{t}\right\|_{C_{*}^{2}}$. By (29) and (30) we see that $\left|\phi_{t}\right| \leq C \varepsilon^{2}$; hence standard elliptic theory [10, Chapter 6] gives

$$
\left\|\phi_{t}\right\|_{C^{2}\left(B_{\varepsilon}\right)} \leq C\left(\left\|\rho_{t}\right\|_{C^{\alpha}\left(B_{1}\right)}+\left\|\phi_{t}\right\|_{B_{1}}\right) \leq \varepsilon^{-\alpha} \text {. }
$$

This, together with (29), gives

$$
\left\|\phi_{t}\right\|_{C_{*}^{2}, B_{6 \varepsilon}}=\left\|\phi_{t}\right\|_{C^{0}, B_{6 \varepsilon}}+\left\|r D \phi_{t}\right\|_{C^{0}, B_{6 \varepsilon}}+\left\|r^{2} D^{2} \phi_{t}\right\|_{C^{0}, B_{6 \varepsilon}} \leq C \varepsilon^{1-\alpha} .
$$


On $\mathbb{R}^{3} \backslash B_{6 \varepsilon}$ we use Lemma 10 and (28), together with the fact that $\rho_{t}$ is bounded, to estimate:

$$
\begin{gathered}
\left|\partial_{i} \phi_{t}(x)\right| \leq C \int_{B_{4 \varepsilon}}\left|\partial_{i} G(x, y)\right| d y \leq C \frac{\varepsilon^{3}}{|x|^{2}} \\
\left|\partial_{i} \partial_{j} \phi_{t}(x)\right| \leq C \int_{B_{4 \varepsilon}}\left|\partial_{i} \partial_{j} G(x, y)\right| d y \leq C \frac{\varepsilon^{3}}{|x|^{3}},
\end{gathered}
$$

which together with (30) yields

$$
\left\|\phi_{t}\right\|_{C_{*}^{2}, \mathbb{R}^{3} \backslash B_{6 \varepsilon}} \leq C \varepsilon
$$

From (31) and (32) we get $\left\|\phi_{t}\right\|_{C_{*}^{2}} \leq C \epsilon^{1-\alpha}$.

Finally, we show that given $g \in C^{\infty} \cap \mathcal{M}$, we can assume without loss of generality that the standard coordinates of $\mathbb{R}^{3}$ are normal coordinates in a neighborhood of the origin. Let $\tilde{F}: B_{R} \rightarrow \mathbb{R}^{3}$ be a $C^{\infty}$ map with $\tilde{F}(0)=0$, $D \tilde{F}_{0}=I$, and for which the standard coordinates $\left(x^{1}, x^{2}, x^{3}\right)$ are normal coordinates with respect to the metric $\tilde{F}^{*} g$ on $B_{R}$. For $0<\epsilon<R / 2$, define

$$
F=\left(1-\eta_{2 \epsilon}\right) \tilde{F}+\eta_{2 \epsilon} I
$$

It is straightforward to check that

$$
\epsilon^{-1}|D F-I|+\left|D^{2} F\right|+\epsilon\left|D^{3} F\right| \leq \frac{C}{\epsilon},
$$

where $C$ is independent of $\epsilon$. In particular, $F$ is a diffeomorphism provided $\epsilon>0$ is small enough. Furthermore, since

$$
\left(F^{*} g\right)_{i j}=g_{k l} \frac{\partial F^{k}}{\partial y^{i}} \frac{\partial F^{l}}{\partial y^{j}}
$$

It follows that $\left\|F^{*} g-g\right\|_{C_{*}^{2}}<C \epsilon^{2}$; hence by Proposition 7 the coordinate spheres are quasiconvex provided $\epsilon>0$ is small. Thus, we may replace $g$ by $F^{*} g$ since they are related by a diffeomorphism $F \in \mathcal{G}$. We have proved

Proposition 12. Let $g_{1} \in \mathcal{M} \cap\left(\delta+C_{-1}^{\infty}\right)$ be harmonically flat. Then there is metric $g_{1}^{\prime}$, diffeomorphic to $g_{1}$, and a continuous path $g_{t} \in \mathcal{M} \cap\left(\delta+C_{-1}^{\infty}\right)$, $1 \leq t \leq 2$, from $g_{1}^{\prime}$ to a harmonically flat metric $g_{2} \in \mathcal{M} \cap\left(\delta+C_{-1}^{\infty}\right)$ that is conformally flat in a neighborhood of the origin. 


\section{Bernoulli-type Parabolic PDEs on $\mathbb{S}^{2}$.}

In this section we collect the analytical results we need to prove the Main Theorem: (conditions for) global existence, uniqueness, asymptotic behavior and continuous dependence on parameters for solutions of (4). All these rely on a simple pointwise a priori bound whose proof we present here. Many of the results presented in this section are adapted from [2]. In order to ensure the uniform parabolicity of (4) we assume throughout this section that $\Gamma=e^{-2 v} / \bar{H}$ is bounded above and below by positive constants.

First, we define parabolic Banach spaces to be used in our study of (4). Let $0<r_{0}<r_{1} \leq \infty, I=\left[r_{0}, r_{1}\right] \subset \mathbb{R}^{+}$, and let $A_{I}=I \times \mathbb{S}^{2}$. Given a function $f$ on $A_{I}$, define:

$$
\begin{gathered}
{[f]_{\alpha ; I}=\sup _{\substack{\left(r_{1}, \theta_{1}\right),\left(r_{2}, \theta_{2}\right) \in A_{I} \\
\operatorname{dist}\left(\theta_{1}, \theta_{2}\right)<\pi}}\left[\frac{\left|f\left(r_{2}, \theta_{2}\right)-f\left(r_{1}, \theta_{1}\right)\right|}{\left|1-r_{2} / r_{1}\right|^{\alpha / 2}+\operatorname{dist}\left(\theta_{2}, \theta_{1}\right)^{\alpha}}\right]} \\
\|f\|_{0 ; I}=\sup _{A_{I}}|f|, \quad\|f\|_{0, \alpha ; I}=\|f\|_{0 ; I}+[f]_{\alpha ; I} .
\end{gathered}
$$

Here $\operatorname{dist}(\cdot, \cdot)$ denotes the geodesic distance on $\mathbb{S}^{2}$. If $f(r, \cdot)$ is a tensor field on $\mathbb{S}^{2}$, then $f\left(r_{2}, \theta_{2}\right)$ is understood to mean the parallel translate of $f\left(r_{2}, \theta_{2}\right)$ back to $\theta_{1}$ along the unique geodesic from $\theta_{1}$ to $\theta_{2}$. With these conventions we can now define:

$$
\|f\|_{k, \alpha ; I}=\sum_{i+2 j \leq k}\left\|\not \not^{i}\left(r \partial_{r}\right)^{j} f\right\|_{0 ; I}+\sum_{i+2 j=k}\left[\not^{i}\left(r \partial_{r}\right)^{j} f\right]_{\alpha ; I},
$$

where $\not^{i} f$ is to be interpreted as the $i$-th covariant derivative of $f$ in the standard metric on $\mathbb{S}^{2}$. Now define $H_{I}^{k, \alpha}$ to be the space of functions $f$ on $A_{I}$ for which $\|f\|_{k, \alpha ; I}$ is defined and finite. Equipped with the norm $\|\cdot\|_{k, \alpha ; I}$, the space $H_{I}^{k, \alpha}$ is a Banach space.

It should be noted that Equation (4) has the following scale invariance: Let $\lambda>0$ and let $I$ be an interval. Given a function $f$ on $A_{\lambda I}$, define on $A_{I}$ the function $f_{\lambda}(r, \theta)=f(\lambda r, \theta)$. If $u$ is a solution of Equation (4) on the scaled interval $\lambda I$ then $u_{\lambda}$ is a solution of (4) on $I$ with the scaled coefficients $A_{\lambda}, B_{\lambda}, \beta_{\lambda}, \Gamma_{\lambda}$. The norms $\|\cdot\|_{k, \alpha ; I}$ are chosen to have the same scale invariance: $\left\|u_{\lambda}\right\|_{k, \alpha ; I}=\|u\|_{k, \alpha ; \lambda I}$.

Following Bartnik in [2], we also use the notation:

$$
f^{*}(r)=\sup _{\theta \in \mathbb{S}^{2}} f(r, \theta), \quad f_{*}(r)=\inf _{\theta \in \mathbb{S}^{2}} f(r, \theta) .
$$




\subsection{Conditions for Global Existence and Uniqueness.}

Our first observation is that Equation (4) is uniformly parabolic with $r$ as the "time" variable provided $u$ is bounded above and below by positive constants. Therefore, given any initial data $u\left(r_{0}, \theta\right)=u_{0}(\theta)$ it is standard to obtain the existence of a unique solution on a short time interval $\left[r_{0}, r_{0}+\epsilon\right)$ for some $\epsilon>0$. Furthermore, it is well known that, for some choices of coefficients and initial data, a classical solution can blow-up in finite time. Thus, our main objective here is to derive conditions that guarantee the existence of a global positive solution on the time interval $\left[r_{0}, \infty\right]$. As seen in the proof of Theorem 14 below, if the coefficients have sufficient regularity then a pointwise a priori bound on a solution $u$ is all that is needed to ensure

global existence. We establish these bounds through the use of upper and lower solutions. Given a parabolic linear operator

$$
F(w)=\left(r \partial_{r}-P \cdot \nabla-Q \not \mathcal{A}+A\right) w+B
$$

acting on functions $w$ on $I \times \mathbb{S}^{2}$, recall that upper and lower solutions, $w_{u}$ and $w_{l}$ respectively, of the equation

$$
F(w)=0,\left.w\right|_{r_{0} \times \mathbb{S}^{2}}=w\left(r_{0}\right)
$$

are functions satisfying $w_{l}\left(r_{0}\right) \leq w\left(r_{0}\right) \leq w_{u}\left(r_{0}\right)$ and

$$
F\left(w_{u}\right) \geq 0, \quad F\left(w_{l}\right) \leq 0
$$

One has the following maximum principle:

Lemma 13. If $w_{u}, w_{l}$ are upper and lower solutions, respectively, of equation (33) then $w_{l} \leq w \leq w_{u}$.

This is essentially [15, Theorem 2.10], but the proof here is simpler since there is no spatial boundary.

Theorem 14. Let $I=\left[r_{0}, \infty\right)$, and suppose that $\beta, \Gamma, r(2 A-1), B \in H_{I}^{k, \alpha}$. Suppose also that $B \geq \sigma_{1}$ for some positive constant $\sigma_{1}$. If $u_{0}>0$ satisfies $u_{0} \in C^{k+2, \alpha}\left(\mathbb{S}^{2}\right)$ then there exists a unique solution $u \in H_{I}^{k+2, \alpha}$ of (4) with initial condition $u\left(r_{0}, \theta\right)=u_{0}(\theta)$. Furthermore, there exists $\sigma_{2}>0$ such that $u>\sigma_{2}$.

Proof. The local in time existence theory gives a positive solution $u \in$ $H_{\left[r_{0}, r_{0}+\varepsilon\right)}^{2, \alpha}$ of Equation (4) with the given initial data for some $\varepsilon>0$. Let $r_{1}$ 
be the supremum of all $r$ for which the solution exists on $\left[r_{0}, r\right)$ and define $I=\left[r_{0}, r_{1}\right)$. On any interval $\left[r_{0}, r\right)$ with $r<r_{1}$, the solution $u$ is the only solution with the given initial data. This is easily seen since the difference of any two such solutions $v=u_{1}-u_{2}$ verifies a linear equation of the form

$$
r \partial_{r} v=u_{1} \Gamma \not \Delta v+\tilde{A} v,\left.\quad v\right|_{r_{0}} \equiv 0,
$$

whose only solution is $v \equiv 0$.

Before we can prove global existence, we establish a supremum bound for $u$ on the interval $I$. As in [2], we find this bound via the auxiliary function $w=u^{-2}$, which satisfies the equation

$$
r \partial_{r} w+P \cdot \not \downarrow w-Q \not \not w+2 A w-2 B=0, \quad w\left(r_{0}\right)=u^{-2}\left(r_{0}\right),
$$

where $P=3 / 2 w^{-2} \not \downarrow w-\beta$ and $Q=\Gamma / w$. Define radial functions $w_{u}, w_{l}$ to be the solutions of the ordinary differential equations

$$
r \frac{d}{d r} w_{l}+2 A^{*} w_{l}-2 B_{*}=0, \quad w_{l}\left(r_{0}\right)=w_{*}\left(r_{0}\right)
$$

and

$$
r \frac{d}{d r} w_{u}+2 A_{*} w_{u}-2 B^{*}=0, \quad w_{u}\left(r_{0}\right)=w^{*}\left(r_{0}\right) .
$$

Since $B>0$ it follows that $w_{u}$ and $w_{l}$ are positive. Thus

$$
r \partial_{r} w_{l}+P \cdot \not \nabla w_{l}-Q \not \not w_{l}+2 A w_{l}-2 B=2\left(A-A^{*}\right) w_{l}-2\left(B-B_{*}\right) \leq 0,
$$

and hence $w_{l}$ is a lower solution. It is similarly verified that $w_{u}$ is an upper solution. Integrating, we find

$$
w_{l}(r)=\frac{1}{r} e^{\int_{r_{0}}^{r} 1-2 A^{*} d r^{\prime} / r^{\prime}}\left(r_{0} w_{*}\left(r_{0}\right)+\int_{r_{0}}^{r} B_{*} e^{\int_{r_{0}}^{r^{\prime}} 2 A^{*}-1 d r^{\prime \prime} / r^{\prime \prime}} d r^{\prime}\right) .
$$

and

$$
w_{u}(r)=\frac{1}{r} e^{\int_{r_{0}}^{r} 1-2 A_{*} d r^{\prime} / r^{\prime}}\left(r_{0} w^{*}\left(r_{0}\right)+\int_{r_{0}}^{r} B^{*} e^{\int_{r_{0}}^{r^{\prime}} 2 A_{*}-1 d r^{\prime \prime} / r^{\prime \prime}} d r^{\prime}\right) .
$$

Using $r\left|2 A_{*}-1\right|, r\left|2 A^{*}-1\right| \leq\|r(2 A-1)\|_{0 ; I},|B|^{*} \leq\|B\|_{0 ; I}$ and $\sigma_{1}$ to estimate these integrals and then applying Lemma 13, it follows that there is a constant $c>0$, not depending on the length of $I$, such that

$$
c^{1 / 2}<w_{l} \leq w \leq w_{u}<c^{-1 / 2} .
$$


Thus, we have

$$
c^{-1}<u<c .
$$

To see that $r_{1}=\infty$, suppose instead that $r_{1}<\infty$. Put $I^{\prime}=\left[r_{0}+\varepsilon / 2, r_{1}\right)$. In divergence form, Equation (4) reads:

$$
r \partial_{r} u=\not b \cdot\left(\tilde{\Gamma} u^{2} \not \mathbf{b} u\right)+\left(\beta-u^{2} \not \tilde{\Gamma}\right) \cdot \nabla u-\left.2 \tilde{\Gamma} u|\not| u\right|^{2}+A u-B u^{3} .
$$

Thus, the conditions (6.30a)-(6.30c) in [15] hold and hence there exist $\alpha^{\prime}, C$ such that $\|u\|_{\alpha^{\prime} ; I^{\prime}} \leq C$; see [15, Theorem 6.28]. We can now regard Equation (4) as a linear equation with coefficients in $H_{I}^{\alpha^{\prime}}$. The standard linear Schauder interior estimates give

$$
\|u\|_{2, \alpha^{\prime} ;\left[r_{0}+\varepsilon, r_{1}\right]} \leq C\|u\|_{0 ; I} \leq C \cdot c ;
$$

see [15, Theorem 4.9]. In particular, for $0<\alpha^{\prime \prime}<\alpha^{\prime}$, the solution $u$ can be extended to a positive function $u \in H_{\left[r_{0}, r_{1}\right]}^{2, \alpha^{\prime \prime}}$. Applying the local existence theory again with initial data $\left.u\right|_{r_{1}}$, the solution can be extended to $\left[r_{0}, r_{1}+\varepsilon^{\prime}\right)$ for some $\varepsilon^{\prime}>0$, in contradiction to the fact that $\left[r_{0}, r_{1}\right)$ is the maximal interval of existence. We conclude that $r_{1}=\infty$.

Finally, we show that $u \in H_{I}^{k+2, \alpha}$. Note first that since the supremum estimates above do not depend on the length of the interval, the bound (35) remains valid on $I=\left[r_{0}, \infty\right)$. Let $I^{\prime}=\left[r_{0}, 4 r_{0}\right]$ and let $I^{\prime \prime}=\left[2 r_{0}, 4 r_{0}\right]$. For $\lambda>1$, let $u_{\lambda}, A_{\lambda}, B_{\lambda}, \Gamma_{\lambda}, \beta_{\lambda}$ be defined by scaling $u, A, B, \Gamma, \beta$ as in the introduction to this section. Then on $I^{\prime}$ the function $u_{\lambda}$ satisfies Equation (4) with the coefficients $A_{\lambda}, B_{\lambda}, \Gamma_{\lambda}, \beta_{\lambda}$. Applying the interior estimates of the previous paragraph followed by the usual bootstrap argument we then obtain $\left\|u_{\lambda}\right\|_{k+2, \alpha ; I^{\prime \prime}} \leq C$, with $C$ not depending on $\lambda$. Thus, by the scale invariance of the $H_{I}^{k, \alpha}$ norms we get $\|u\|_{k+2, \alpha ; \lambda I^{\prime \prime}} \leq C$. Taking the supremum over $\lambda>1$ yields $\|u\|_{k+2, \alpha ;\left[2 r_{0}, \infty\right)} \leq C$. It remains to bound $\|u\|_{k+2, \alpha ;\left[r_{0}, 2 r_{0}\right]}$. From the previous paragraph we already have $\|u\|_{2, \alpha^{\prime} ;\left[r_{0}, 2 r_{0}\right]} \leq C$. The bound $\|u\|_{k+2, \alpha ;\left[r_{0}, 2 r_{0}\right]} \leq C$ now follows from the usual bootstrap argument using boundary type estimates; see [15, Theorem 4.28].

We will also need global existence when $B<0$ somewhere. To obtain the necessary bounds from scratch requires conditions on the initial data, see [2]. To avoid having to control the initial data we will instead use the following comparison result:

Theorem 15. Let $I=\left[r_{0}, \infty\right)$, and suppose that $u>0$ is a classical solution of Equation (4) on $I \times \mathbb{S}^{2}$ with coefficients $\beta, \Gamma, r(2 A-1), B \in H_{I}^{k, \alpha}$ and 
with initial data $u_{0} \in C^{k+2, \alpha}\left(\mathbb{S}^{2}\right)$. Let $\tilde{B} \in H_{I}^{k, \alpha}$ satisfy $\tilde{B} \geq B$. Then the equation

$$
r \partial_{r} u-\beta \cdot \not \nabla u=\Gamma u^{2} \not \mathbf{X} u+A u-\tilde{B} u^{3}
$$

has a unique solution $\tilde{u} \in H_{I}^{k+2, \alpha}$ with the same initial data $\tilde{u}\left(r_{0}, \theta\right)=u_{0}(\theta)$. Furthermore, for some $\sigma>0$ we have $\sigma<\tilde{u} \leq u$.

Proof. If we can verify a bound of the form (35) on any interval $\left[r_{0}, r\right)$ for which the solution exists and is positive then, as in the proof of the previous theorem, global existence of a unique positive solution $u \in H_{I}^{k+2, \alpha}$ will follow.

Subtracting the equation for $\tilde{u}$ from the equation for $u$ we get an equation for $v=u-\tilde{u}$ :

$$
r \partial_{r} v-\beta \cdot \not \downarrow v=\Gamma u^{2} \not \Delta v+\tilde{A} v-(B-\tilde{B}) u^{3},
$$

where $\tilde{A}=\Gamma(\tilde{u}+u) \not \alpha \tilde{u}-\tilde{B}\left(\tilde{u}^{2}+\tilde{u} u+u^{2}\right)$. Since $\tilde{B}-B>0$ it is clear that $v_{l} \equiv 0$ is a lower solution of this equation and so by lemma 13 we see that $\tilde{u} \leq u$. To bound $u$ from below we use the auxiliary function $w$ defined in the proof of Theorem 14. In this case, however, we take the solution of

$$
r \frac{d}{d r} w_{u}+2 A_{*} w_{u}-2|B|^{*}=0, \quad w_{u}\left(r_{0}\right)=w^{*}\left(r_{0}\right)
$$

as an upper solution for Equation (34). Continuing the argument as in the proof of Theorem 14 we find a positive constant $\sigma$ such that $u>\sigma$.

For the remainder of this section we set $I=\left[r_{0}, \infty\right)$.

\subsection{Asymptotic Behavior.}

To study the asymptotic behavior of a given solution $u$ of Equation (4),we define $m$ by $u^{-2}=1-2 m / r$. Then $m$ verifies on $I \times \mathbb{S}^{2}$ the equation:

$$
r \partial_{r} m-\left(\beta+\frac{3 \Gamma u^{4}}{r} \not \nabla m\right) \cdot \not \downarrow m=\Gamma u^{2} \not \Delta m-(2 A-1) m+r(A-B)
$$

This equation has a useful scale covariance: The function $\lambda^{-1} m_{\lambda}$ satisfies the above equation when the coefficients are replaced by $u_{\lambda}, A_{\lambda}, B_{\lambda}, \Gamma_{\lambda}, \beta_{\lambda}$. We have:

Theorem 16. Let $\beta, \Gamma, r(2 A-1), r(A-B) \in H_{I}^{k, \alpha}$, and let $u \in H_{I}^{k+2, \alpha}$ be a solution of Equation (4) satisfying $u>\sigma$ for a positive constant $\sigma$. If in addition $|A-B|^{*} \in L^{1}(I)$, then $m=r\left(1-u^{-2}\right) / 2 \in H_{I}^{k+2, \alpha}$. 
Proof. Define $I^{\prime}=\left[r_{0}, 4 r_{0}\right]$ and $I^{\prime \prime}=\left[2 r_{0}, 4 r_{0}\right]$. By the definition of $m$ it is clear that $\|m\|_{k+2, \alpha ; I^{\prime}}$ is bounded by a constant only depending on $\|u\|_{k+2, \alpha ; I}$, $r_{0}$, and $\sigma$. In view of the scaling properties of Equation (37), the standard Schauder interior estimates give, as above,

$$
\lambda^{-1}\left\|m_{\lambda}\right\|_{k+2, \alpha ; I^{\prime \prime}} \leq C\left(\lambda^{-1}\|m\|_{0, I^{\prime}}+\left\|r\left(A_{\lambda}-B_{\lambda}\right)\right\|_{k, \alpha ; I^{\prime}}\right)
$$

for any $\lambda>1$. By the scale invariance of the $H_{I}^{k, \alpha}$ norms we have

$$
\|m\|_{k+2, \alpha ; \lambda I^{\prime \prime}} \leq C\left(\|m\|_{0, \lambda I^{\prime}}+\|r(A-B)\|_{k, \alpha ; \lambda I^{\prime}}\right),
$$

where $C$ does not depend on $\lambda$. Thus, the theorem will follow if we can establish a bound on $\sup |m|$.

Define $v$ to be the radial function satisfying

$$
r \frac{d}{d r} v=|2 A-1|^{*} v+r|A-B|^{*}, \quad v\left(r_{0}\right)=\left|m\left(r_{0}\right)\right|^{*}
$$

Then $v$ remains positive and thus it is an upper solution of Equation (37) with the initial data $m\left(r_{0}\right)$. Integrating this equation and applying Lemma 13 yields the upper bound for $m$

$$
m \leq e^{\int_{r_{0}}^{r_{1}}|2 A-1|^{*} d r / r}\left(\left|m\left(r_{0}\right)\right|^{*}+\int_{r_{0}}^{\infty}|A-B|^{*} e^{\int_{r_{0}}^{r}|2 A-1|^{*} d r^{\prime} / r^{\prime}} d r\right) .
$$

Similarly, we have

$$
m \geq-e^{\int_{r_{0}}^{r_{1}}|2 A-1|^{*} d r / r}\left(\left|m\left(r_{0}\right)\right|^{*}+\int_{r_{0}}^{\infty}|A-B|^{*} e^{\int_{r_{0}}^{r}|2 A-1|^{*} d r^{\prime} / r^{\prime}} d r\right) .
$$

Since $|A-B|^{*} \in L^{1}$ it follows that $|m|$ is uniformly bounded.

We will also need $m \in H_{I}^{k+2, \alpha}$ in some cases when the condition $|A-B|^{*} \in$ $L^{1}(I)$ cannot be verified. To this end, we state a comparison result that establishes a bound on $m$. Let $u_{i}, i=1,2$, satisfy

$$
r \partial_{r} u_{i}-\beta \cdot \not u_{i}=\Gamma u_{i}^{2} \not u_{i}+A u_{i}-B_{i} u_{i}^{3}
$$

Setting $m_{i}=r\left(1-u_{i}^{-2}\right) / 2$, and $\tilde{m}=m_{2}-m_{1}$, we obtain, in view of (37)

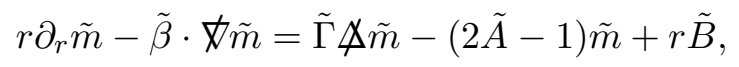


where

$$
\begin{aligned}
\tilde{\beta} & =\beta+3 r^{-1} \Gamma u_{2}^{4} \not\left(m_{1}+m_{2}\right) \\
\tilde{A} & =A-\Gamma u_{1}^{2} u_{2}^{2}\left(r^{-1} \not m_{1}+3 r^{-2}\left(u_{1}^{2}+u_{2}^{2}\right)\left|\not \supset m_{1}\right|^{2}\right) \\
\tilde{\Gamma} & =u_{2}^{2} \Gamma \\
\tilde{B} & =B_{2}-B_{1} .
\end{aligned}
$$

Considering this equation for the difference allows us to circumvent the condition that $|A-B|^{*}$ be $L^{1}$ in the case that we have at least one solution of Equation (37) with the desired asymptotic behavior.

Theorem 17. Let $\beta_{1}, \Gamma, r(2 A-1), B_{1}, B_{2} \in H_{I}^{k, \alpha}$. With $i=1,2$, suppose that $u_{i} \in H_{I}^{k+2, \alpha}$ are positive solutions of Equation (38). If $\left|B_{1}-B_{2}\right|^{*} \in$ $L^{1}(I)$ and $m_{1} \in H_{I}^{k+2, \alpha}$ then $m_{2}=r\left(1-u_{2}^{-2}\right) / 2 \in H_{I}^{k+2, \alpha}$.

Proof. Note that $m_{1}, u_{1}, u_{2} \in H_{I}^{k+2, \alpha}$ and $\not \nabla m_{1}, \not \Delta m_{1} \in H_{I}^{k, \alpha}$. Thus, the coefficients $\tilde{A}, \tilde{\Gamma}, \tilde{\beta}, \tilde{B}$ satisfy the same hypotheses as $A, \Gamma, \beta, A-B$ in the previous theorem, which then applies to give $\tilde{m} \in H_{I}^{k+2, \alpha}$. Clearly, $m_{2}=$ $m_{1}+\tilde{m} \in H_{I}^{k+2, \alpha}$.

\subsection{Continuous Dependence on Parameters.}

In this subsection we prove the continuous dependence on parameters of solutions of Equations (4) and (37). Since every solution $u$ of Equation (4) is related to a solution $m$ of Equation (37) by $u^{-2}=1-2 m / r$, it suffices to prove the continuous dependence of solutions to Equation (37). We do this by using the implicit function theorem, but this requires us to restrict the domain and range of the linear parabolic operator

$$
T(v) \equiv \partial_{r} v+\frac{P}{r} \cdot \not \downarrow v-\frac{Q}{r} \not \mathbf{X} v+\frac{\tilde{A}}{r} v
$$

so that it is an isomorphism. With $T_{0} \equiv \partial_{r}-(2 r)^{-1} \not \mathbf{X}$ and $\|f\|_{L^{1, *}} \equiv \int_{I}|f|^{*}$, define

$$
\begin{aligned}
X_{I}^{k, \alpha} & =\left\{v \in H_{I}^{k, \alpha}: v\left(r_{0}\right)=0,\left|T_{0} v\right|^{*} \in L^{1}(I)\right\}, \\
\|v\|_{X_{I}^{k, \alpha}} & =\|v\|_{I, k ; \alpha}+\left\|T_{0} v\right\|_{L^{1, *}}
\end{aligned}
$$


and

$$
\begin{aligned}
Y_{I}^{k, \alpha} & =\left\{v \in r^{-1} H_{I}^{k, \alpha}:|v|^{*} \in L^{1}(I)\right\}, \\
\|v\|_{Y_{I}^{k, \alpha}} & =\|r v\|_{I, k ; \alpha}+\|v\|_{L^{1, *}} .
\end{aligned}
$$

It is easy to check that $X_{I}^{k, \alpha}$ and $Y_{I}^{k, \alpha}$ are Banach spaces.

We have:

Lemma 18. If $r P, r(Q-1 / 2), r \tilde{A} \in H_{I}^{k, \alpha}$ then $T: X_{I}^{k+2, \alpha} \rightarrow Y_{I}^{k, \alpha}$ is an isomorphism.

Proof. Writing

$$
T(v)=T_{0} v+\frac{1}{r}\left(\frac{1}{2}-Q\right) \not \Delta v+\frac{P}{r} \cdot \not \nabla v+\frac{\tilde{A}}{r} v
$$

it is apparent from the definition of $X_{I}^{k+2, \alpha}$ that $T$ maps this space to $Y_{I}^{k, \alpha}$. Also, given $f \in Y_{I}^{k, \alpha}$, it is clear that there is a classical solution $v$ of

$$
T(v)=f, \quad v\left(r_{0}\right)=0 .
$$

It must be shown that $v \in X_{I}^{k+2, \alpha}$. Upper and lower solutions are used as in the proof of Theorem 16 to obtain a bound $\|v\|_{H_{I}^{0}} \leq C\|f\|_{L^{1, *}}$, where $C$ depends only on $\|r P\|_{I, k, \alpha},\|r(1 / 2-Q)\|_{I, k, \alpha},\|r A\|_{I, k, \alpha}$. Noting that the the equation $T(v)=f$ has the same scaling properties as the equation for $m$ above, we apply the Schauder theory as in the proof of Theorem 16 to find

$$
\|v\|_{k+2, \alpha,\left[\lambda r_{0}^{\prime}, \lambda r_{1}^{\prime}\right]} \leq C\|f\|_{Y_{I}^{k, \alpha}}
$$

where $\lambda>1$, and $C$ does not depend on $\lambda$. Using then boundary type estimates near $r_{0}$ and taking the supremum over $\lambda$ yields

$$
\|v\|_{k+2, \alpha ; I} \leq C\|f\|_{Y_{I}^{k, \alpha}} .
$$

Finally, noting that

$$
\left|T_{0} v\right|^{*}=\left|\frac{1}{r}\left(\frac{1}{2}-Q\right) \not \Delta v-\frac{P}{r} \cdot \not \downarrow v-\frac{\tilde{A}}{r} v-f\right|^{*} \leq \frac{\|v\|_{k+2, \alpha ; I}}{r^{2}},
$$

it follows that $\|v\|_{X_{I}^{k+2, \alpha}} \leq C\|f\|_{Y_{I}^{k, \alpha}}$ and the lemma is proved. 
Theorem 19. Let $I=\left[r_{0}, \infty\right)$, and let $J=[a, b]$. Suppose that $u$ is a family of solutions of Equation (4) with the coefficients $\beta, \Gamma, A, B$ satisfying $r(2 A-1), r(2 B-1), r \beta, r(\Gamma-1 / 2) \in C^{0}\left([a, b], H_{I}^{k, \alpha}\right)$ and with the initial data $u\left(r_{0}\right) \in C^{0}\left(J, C^{k+2, \alpha}\left(\mathbb{S}^{2}\right)\right)$. Suppose either of the following two conditions is satisfied:

(i) $|A-B|^{*} \in C^{0}\left(J, L^{1}(I)\right)$.

(ii) A family of solutions $m^{\prime} \in C^{0}\left([a, b], H_{I}^{k, \alpha}\right)$ exists for Equation (37) with $B$ replaced by some $B^{\prime}$ satisfying $r\left(2 B^{\prime}-1\right) \in C^{0}\left([a, b], H_{I}^{k, \alpha}\right)$.

Then $u, m \in C^{0}\left(J, H_{I}^{k, \alpha}\right)$.

Proof. First of all, note that Conditions (i) and (ii) guarantee that the family of solutions $m$ will satisfy $m \in H_{I}^{k+2, \alpha}$ for every value of the parameter. When Condition (i) is satisfied this follows from Theorem 16 and when Condition (ii) is satisfied this follows from Theorem 17.

Define $H_{-1 ; I}^{k, \alpha}$ to consist of those elements $v$ of $H_{I}^{k, \alpha}$ for which $r v \in H_{I}^{k, \alpha}$ also. Define $\|v\|_{H_{-1 ; I}^{k, \alpha}}=\|r v\|_{k, \alpha, I}$. Define

$$
W=C^{k+2, \alpha}\left(\mathbb{S}^{2}\right) \times H_{-1 ; I}^{k, \alpha} \times H_{-1 ; I}^{k, \alpha} \times H_{-1 ; I}^{k, \alpha} \times Y_{I}^{k, \alpha} .
$$

Let $m=v+m\left(r_{0}\right)$. Define the operator $F: W \times X_{I}^{k+2, \alpha} \rightarrow Y_{I}^{k, \alpha}$ for $w=\left(m\left(r_{0}\right), \beta, \Gamma-1 / 2, A^{\prime}, B^{\prime}\right)$ by

$$
\begin{aligned}
F(w, v)=\partial_{r} m-\frac{\Gamma}{r} & \left(1-\frac{2 m}{r}\right)^{-1} \not \Delta m \\
& -\left(\frac{\beta}{r}+\frac{3 \Gamma}{r^{2}}\left(1-\frac{2 m}{r}\right)^{-2} \not \triangleright m\right) \cdot \not 內 m+\frac{A^{\prime}}{r} m-B^{\prime}
\end{aligned}
$$

Note that $F$ is differentiable in $w$ and $m$. We have

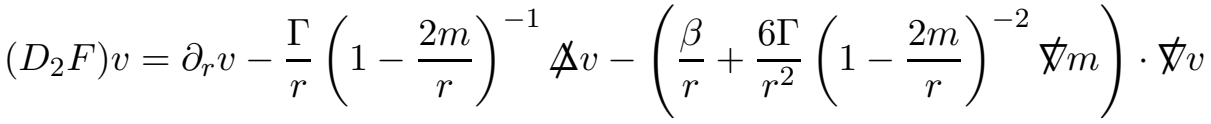

$$
\begin{aligned}
& -\left(\frac{A^{\prime}}{r}+\frac{2 \Gamma}{r^{2}}\left(1-\frac{2 m}{r}\right)^{-2} \not \Delta m+\frac{12 \Gamma}{r^{3}}\left(1-\frac{2 m}{r}\right)^{-3}|\not m m|^{2}\right) v \text {, }
\end{aligned}
$$

which by Lemma 18 is an isomorphism from $X_{I}^{k, \alpha}$ into $Y_{I}^{k, \alpha}$ when $m \in H_{I}^{k, \alpha}$, $2 m<r$. Given $v_{0}<r / 2-m\left(r_{0}\right)$ and $w_{0}$ such that $F\left(w_{0}, v_{0}\right)=0$, it follows 
then from the implicit function theorem that there is a neighborhood $M$ of $w_{0}$ such that there is a uniquely defined differentiable function $f: M \rightarrow$ $X_{I}^{k, \alpha}$ with $f\left(w_{0}\right)=v_{0}$. In particular, for $w \in W$ the unique solution of $F(w, v)=0$ is continuous in $w$. Since Equation (37) can be written as $F(w, v)=0$ with $w=\left(m\left(r_{0}\right), \beta, \Gamma, 2 A-1, B-A\right)$, it follows that if $u, m$ are as in the hypothesis of the theorem when Condition (i) is satisfied we have that $u, m \in C^{0}\left(J, H_{I}^{k, \alpha}\right)$.

For the case that Condition (ii) is satisfied, put $B_{1}=B^{\prime}, B_{2}=B$, and $m_{1}=m^{\prime}$ so that the difference $\tilde{m}=m-m^{\prime}$ verifies Equation (39) above. We now use this equation to define the operator $\tilde{F}$ in the same way that we used Equation (37) to define $F$. Applying the argument of the previous paragraph then shows that $\tilde{m} \in C^{0}\left(J, H_{I}^{k, \alpha}\right)$ and so $m=m^{\prime}+\tilde{m} \in C^{0}\left(J, H_{I}^{k, \alpha}\right)$.

\section{Deformation.}

We are now in a position to prove the main theorem. We begin by restricting attention to a subset $\mathcal{M}_{1} \subset \mathcal{M}$ defined as follows: $\mathcal{M}_{1}$ consists of all harmonically flat metrics $g \in \mathcal{M} \cap C_{-1}^{\infty}$ that in addition are conformal to $\delta$ on a neighborhood of the origin. The next proposition, which follows directly from Propositions 11 and 12 of Section 3, shows that attention can be restricted to $\mathcal{M}_{1}$ without loss of generality.

Proposition 20. For any $g \in \mathcal{M}$, there exists a path of metrics $g_{\lambda} \in \mathcal{M}$, continuous in $C_{-1}^{2, \alpha}$, such that $g_{0}$ is diffeomorphic to $g$, and $g_{1} \in \mathcal{M}_{1}$.

In general, given a topological space $\mathcal{M}^{\prime}$ and a subset $M^{\prime \prime} \subset \mathcal{M}^{\prime}$, we say that $\mathcal{M}^{\prime \prime}$ is connected to $\mathcal{M}^{\prime}$ if for each $g \in \mathcal{M}^{\prime}$ there is a path $g_{\lambda}$ in $\mathcal{M}^{\prime}$ with $g_{0}=g$ and $g_{1} \in \mathcal{M}^{\prime \prime}$. Thus, the previous proposition can be rephrased as $\mathcal{M} / \mathcal{G}$ is connected to $\mathcal{M}_{1}$.

The benefit of working within $\mathcal{M}_{1}$ is that the results of Section 2 imply that any member of $\mathcal{M}_{1}$ is diffeomorphic to a metric $g$ that can be written in the form

$$
g=u^{2} d r^{2}+e^{2 v} \bar{\gamma}_{A B}\left(\beta^{A} d r+r d \theta^{A}\right)\left(\beta^{A} d r+r d \theta^{A}\right),
$$

where $\delta=d r^{2}+r^{2} \bar{\gamma}$ and $u-1, v, \sigma^{-1} \beta \in C_{-1}^{\infty}$. Let $F: \mathbb{R}^{3} \backslash\{0\} \rightarrow \mathbb{R}^{+} \times \mathbb{S}^{2}$ be spherical coordinates as defined in Section 2. We use $F$ to identify $\mathbb{R}^{3} \backslash B_{r_{0}}$ with $I \times \mathbb{S}^{2}$, where $I=\left[r_{0}, \infty\right)$. The following embeddings then hold:

$$
H_{I}^{4 k, 4 \alpha} \subset r C_{-1}^{2 k, 2 \alpha}\left(\mathbb{R}^{3} \backslash B_{r_{0}}\right) \subset H_{I}^{2 k, 2 \alpha}
$$


for functions, and

$$
H_{I}^{4 k, 4 \alpha} \subset C_{-1}^{2 k, 2 \alpha}\left(\mathbb{R}^{3} \backslash B_{r_{0}}\right) \subset H_{I}^{2 k, 2 \alpha}
$$

for one forms $\beta_{A} d \theta^{A}$ tangential to the foliation $\left\{S_{r}\right\}$ of coordinate spheres. Thus $r(u-1), r v, r \beta \in H_{I}^{2 k}$ for every integer $k$.

We define now a nested sequence of subsets $\mathcal{M}=\mathcal{M}_{0} \supset \cdots \supset \mathcal{M}_{4}$, where $\mathcal{M}_{1}$ was defined above and the remaining $\mathcal{M}_{i}$ are given by:

$$
\begin{aligned}
& \mathcal{M}_{2}=\left\{g \in \mathcal{M}_{1}: 2 \kappa-R>0\right\}, \\
& \mathcal{M}_{3}=\left\{g \in \mathcal{M}_{2}: v \text { compactly supported }\right\}, \\
& \mathcal{M}_{4}=\left\{g \in \mathcal{M}_{3}: g \text { is flat }\right\} .
\end{aligned}
$$

The Main Theorem follows by Propositions 21-25 below, which state that each $\mathcal{M}_{i} / \mathcal{G}$ is connected to $\mathcal{M}_{i+1} / \mathcal{G}$ for $i=1,2,3$.

The deformation paths are constructed in the proofs of Propositions 2125 as outlined in the introduction. In these proofs it will be implicitly assumed that $g$ is replaced with an equivalent metric that can be written in the form (40). Thus, for each $g \in \mathcal{M}_{1}$, we can associate corresponding functions $u, v$, and a tangential one-form $\beta$. Note that, by the construction of Section 2, the one form $\beta$ is compactly supported. Let $J=[0,1]$ be the deformation interval. The deformation will be done differently on the regions $B_{r_{0}}$ and $\mathbb{R}^{3} \backslash B_{r_{0}}$. On $B_{r_{0}}$ the path $g_{\lambda}$ is defined directly so that $R\left(g_{\lambda}\right) \geq 0$ and so that the coordinate spheres remain quasi-convex. Furthermore, $u_{\lambda}$, $v_{\lambda}$, and $\beta_{\lambda}$, corresponding to $\left.g_{\lambda}\right|_{B_{r_{0}}}$, satisfy $u_{\lambda}, v_{\lambda}, \beta_{\lambda} \in C^{0}\left(J, C^{k}\left(B_{r_{0}}\right)\right)$ for every integer $k$. In particular, this gives a continuous deformation $u_{\lambda}, v_{\lambda}$, $\beta_{\lambda}$ on the boundary $\partial B_{r_{0}}=S_{r_{0}}$. Next, we define a deformation $v_{\lambda}, \beta_{\lambda}$, $R_{\lambda}$ on $\mathbb{R}^{3} \backslash B_{r_{0}}$, satisfying $v_{\lambda}, r^{-1} \beta_{\lambda}, r^{2} R_{\lambda} \in C^{0}\left(J, C_{-1}^{k}\left(R^{3} \backslash B_{r_{0}}\right)\right)$ so that the normalized mean and Gauss curvatures $\bar{H}_{\lambda}=\bar{H}\left(v_{\lambda}, \beta_{\lambda}\right), \bar{\kappa}_{\lambda}=\bar{\kappa}\left(v_{\lambda}\right)$ remain positive and $R_{\lambda}$ remains nonnegative. It can also be arranged that $v_{\lambda}, \beta_{\lambda}$, and $R_{\lambda}$ are smooth across $\partial B_{r_{0}}$. Taking $u_{\lambda}\left(r_{0}\right)$ corresponding to $\left.g_{\lambda}\right|_{S_{r_{0}}}$ as initial data, we now use the theorems of Section 4 to solve (4) for $u_{\lambda}$ on $\mathbb{R}^{3} \backslash B_{r_{0}}$. At this stage it is checked using Theorem 19 that $m_{\lambda}=2^{-1} r(1-$ $\left.u_{\lambda}^{-2}\right)$ is a continuous family in $H_{I}^{4,2 \alpha}$ so we may conclude $u_{\lambda}-1 \in C^{0}\left(J, C_{-1}^{2, \alpha}\right)$. Substituting $u=u_{\lambda}, v=v_{\lambda}$ and $\beta=\beta_{\lambda}$ in (40) we obtain a continuous deformation $g_{\lambda}$ on $\mathbb{R}^{3} \backslash B_{r_{0}}$ with scalar curvature $R_{\lambda} \geq 0$. Finally, solving (4) starting on an slightly earlier sphere $\partial B_{\left(r_{0}-\varepsilon\right)}$, the uniqueness and regularity results for (4) yield the regularity of $g_{\lambda}$ across $\partial B_{r_{0}}$.

Proposition 21. $\mathcal{M}_{1} / \mathcal{G}$ is connected to $\mathcal{M}_{2} / \mathcal{G}$. 
Proof. Let $g \in \mathcal{M}_{1}$; choose $r_{1}$ such that $2 \kappa-R>0$ for $r<r_{1}$, and choose $r_{0}$ with $r_{0}<r_{1}$. For each $\lambda \in[0,1]$, define $g_{\lambda}=g$ inside $B_{r_{0}}, \beta_{\lambda}=\beta$, and $v_{\lambda}=v$ everywhere. We have $\bar{H}_{\lambda}>0$. Let $\varphi(r)$ be a smooth cut-off function on $[0, \infty)$, satisfying $0 \leq \varphi \leq 1, \varphi=1$ on $\left[0, r_{0}\right]$, and $\varphi=0$ on $\left[r_{1}, \infty\right)$. Define $\varphi_{\lambda}(r)=(1-\lambda)+\lambda \varphi(r)$, and define $R_{\lambda}=\varphi_{\lambda} R$. Then $R_{\lambda}$ is monotonically decreasing in $\lambda, R_{\lambda}=R$ on $B_{r_{0}}$, and $\operatorname{supp}\left(R_{1}\right) \subset B_{r_{1}}$. Thus, Theorems 15 and 17 can be used to solve Equation (4) on $\left[r_{0}, \infty\right) \times \mathbb{S}^{2}$ for $u_{\lambda} \in H^{4, \alpha}$. Also, part (ii) holds in the hypothesis of Theorem 19, which then implies that $u_{\lambda}, m_{\lambda}$ are continuous paths in $H^{4,2 \alpha}$; whence $g_{\lambda} \in C^{0}\left([0,1], C_{-1}^{2, \alpha}\right)$. Clearly, $g_{1} \in \mathcal{M}_{2}$ and the lemma follows.

Remark 22. By applying a fixed diffeomorphism to the path constructed above, it can be shown that in fact $\mathcal{M}_{1}$ is connected to $\mathcal{M}_{2}$.

The final two propositions are proved using blow-down and blow up techniques, respectively. We will need the scaling diffeomorphism: $\phi_{\lambda}:(r, \omega) \mapsto$ $(\lambda r, \omega)$. Note also that in the standard coordinates $x=\left(x^{1}, x^{2}, x^{3}\right)$ of $\mathbb{R}^{3}$ one has $\phi_{\lambda}: x \mapsto \lambda x$. Thus, it follows that $g_{\lambda} \equiv \lambda^{-2} \phi_{\lambda}^{*} g=g_{i j}(\lambda x) d x^{i} d x^{j}$ and $R\left(g_{\lambda}\right)=\lambda^{-2} \phi_{\lambda}^{*} R(g)$, or equivalently $r^{2} R$ is invariant under $g \mapsto g_{\lambda}$. Using the spherical representation of $\phi_{\lambda}$ one finds that if $u, v, \beta$ corresponds to $g$ then $\phi_{\lambda}^{*} u, \phi_{\lambda}^{*} v, \phi_{\lambda}^{*} \beta$ corresponds to $g_{\lambda}$. Using this, and the expressions (13) and (14) of Section 2 for $\bar{H}, \bar{\kappa}$, one has

$$
\begin{aligned}
\phi_{\lambda}^{*} \bar{H} & =\bar{H}\left(g_{\lambda}\right)=\bar{H}\left(v_{\lambda}, \beta_{\lambda}\right) \\
\phi_{\lambda}^{*} \bar{\kappa} & =\bar{\kappa}\left(g_{\lambda}\right)=\bar{\kappa}\left(v_{\lambda}\right) .
\end{aligned}
$$

Finally, we remark that if $g$ has mass function $m=2 r^{-1}\left(1-u^{-2}\right)$ then $g_{\lambda}$ has mass function $\lambda^{-1} m \circ \phi_{\lambda}$. That is

$$
m\left(g_{\lambda}\right)=\lambda^{-1} m \circ \phi_{\lambda}
$$

The following lemma is needed in the next proposition:

Lemma 23. Let $f \in C_{-1}^{k, \alpha}$ be a covariant tensorfield of rank $s$. Define $f_{\lambda}$ by $f_{\lambda}=\lambda^{-s}\left(\phi_{\lambda}\right)_{*} f$. Then $f_{\lambda} \in C^{0}\left([1, \infty), C_{-1}^{k}\right)$, and $\lim _{\lambda \rightarrow \infty}\left\|f_{\lambda}\right\|_{C_{-1}^{0}}=0$.

Proof. It is easy to see that $f_{\lambda}(x)=(f(\lambda x))_{i, j, \ldots, k} d x^{i} d x^{j}, \ldots, d x^{k}$, and thus it is sufficient to prove the theorem for the case that $f$ is a function. 
First, suppose that $k=0$, and let $1 \leq \lambda_{1}<\lambda_{2}$. One has

$$
\begin{gathered}
\sigma(x)\left|f\left(\lambda_{1} x\right)-f\left(\lambda_{2} x\right)\right| \\
=\sigma(x)\left|\lambda_{1}-\lambda_{2}\right|^{\alpha}\left(\frac{r}{\sigma\left(\lambda_{1} x\right)}\right)^{\alpha} \frac{\sigma\left(\lambda_{1} x\right)^{\alpha}\left|f\left(\lambda_{1} x\right)-f\left(\lambda_{2} x\right)\right|}{\left|\lambda_{1} x-\lambda_{2} x\right|^{\alpha}} \\
\leq \frac{\left|\lambda_{1}-\lambda_{2}\right|^{\alpha}}{\lambda_{1}{ }^{1+\alpha}}\|f\|_{C_{-1}^{0, \alpha}} .
\end{gathered}
$$

Taking the supremum over $x$ yields

$$
\left\|f_{\lambda_{1}}-f_{\lambda_{2}}\right\|_{C_{-1}^{0}} \leq \frac{\left|\lambda_{1}-\lambda_{2}\right|^{\alpha}}{\lambda_{1}^{1+\alpha}}\|f\|_{C_{-1}^{0, \alpha}}
$$

from which it follows that $f_{\lambda} \in C^{0}\left([1, \infty), C_{-1}^{0}\right)$. Also,

$$
\left\|f_{\lambda}\right\|_{C_{-1}^{0}}=\sup _{x} \sigma(x)|f(\lambda x)| \leq \lambda^{-1} \sigma(\lambda x)|f(\lambda x)|=\frac{\|f\|_{C_{-1}^{0}}}{\lambda}
$$

and thus $\lim _{\lambda \rightarrow \infty}\left\|f_{\lambda}\right\|_{C_{-1}^{0}}=0$.

In the case that $k>0$, applying the argument of the previous paragraph to the functions $\sigma^{|i|} D^{i} f$ for all multi-indices $|i| \leq k$ proves the lemma.

Proposition 24. $\mathcal{M}_{2} / \mathcal{G}$ is connected to $\mathcal{M}_{3} / \mathcal{G}$.

Proof. Let $g \in \mathcal{M}_{2}$. We take $\lambda \in[1, \infty)$. Assume that $r_{0}$ is large enough that $\operatorname{supp} R \cup \operatorname{supp} \beta \subset B_{r_{0}}$, and put $g_{\lambda}=g$ on $B_{r_{0}}$. Take $R_{\lambda}=R, \beta_{\lambda}=\beta$ everywhere so that $R_{\lambda}=0, \beta_{\lambda}=0$ on $\mathbb{R}^{3} \backslash B_{r_{0}}$. Let $r_{0}<r_{1}$, and define $\tilde{v}_{\lambda}=\left(\phi_{\lambda}\right)_{*} v$ and

$$
v_{\lambda}=\varphi v+(1-\varphi) \tilde{v}_{\lambda},
$$

where $\varphi(r)$ is a cut-off function as in the proof of Proposition 21. Lemma 23 implies that $v_{\lambda} \in C^{0}\left([0, \infty), C_{-1}^{k}\right)$ for all integers $k$, and hence we have $r v_{\lambda} \in C^{0}\left([0, \infty), H_{I}^{4,2 \alpha}\right)$. Using the scaling property (44) and the equation for Gauss curvature (14) we have

$e^{2 v_{\lambda}} \kappa\left(v_{\lambda}\right)=\varphi e^{2 v} \kappa(v)+(1-\varphi) e^{2 \tilde{v}_{\lambda}} \kappa\left(\tilde{v}_{\lambda}\right)=\varphi e^{2 v} \kappa(g)+(1-\varphi) \phi_{\lambda}^{*}\left(e^{2 v} \kappa(g)\right)>0$.

Using the equation for the mean curvature (13), the scaling property (43), and the fact that $\beta=0$ outside $B_{r_{0}}$ we have also on $\mathbb{R}^{3} \backslash B_{r_{0}}$ :

$$
\bar{H}_{\lambda} \equiv \bar{H}\left(v_{\lambda}, \beta_{\lambda}\right)=\varphi H(g)+(1-\varphi) \phi_{\lambda}^{*} H(g)+\left(v-\tilde{v}_{\lambda}\right) r \varphi^{\prime} .
$$


Defining $h=\inf H(g)$ it follows that

$$
\bar{H}_{\lambda} \geq h-\left|\left(v-\tilde{v}_{\lambda}\right) r \varphi^{\prime}\right| \geq h-2\|v\|_{C_{-1}^{0}}\left|\varphi^{\prime}\right| .
$$

Hence, choosing $r_{1}-r_{0}$ large enough one has $\bar{H}_{\lambda}>c>0$. The existence of the family of solutions $u_{\lambda}$ with initial data as given by $\left.g_{\lambda}\right|_{S_{r_{0}}}$ now follows from Theorem 14. Note that outside $B_{r_{1}}$ we have that $v_{\lambda}=\phi_{\lambda}^{*} v, \beta_{\lambda}=\phi_{\lambda}^{*} \beta=0$ correspond to the metric $\lambda^{-2} \phi_{\lambda}^{*} g \in C_{-1}^{\infty}$, and hence the scaling property (45) implies that $\lambda^{-1}\left(\phi_{\lambda}\right)_{*} m$ is a uniformly bounded family of solutions of (37). Theorem 17 then implies $m_{\lambda} \in H^{4,2 \alpha}$ for each $\lambda$. Condition (ii) in the hypothesis of Theorem 19 is now satisfied so that we can conclude $u_{\lambda}, m_{\lambda} \in$ $C^{0}\left([1, \infty), H^{4,2 \alpha}\right)$, and hence $g_{\lambda} \in C^{0}\left([1, \infty), C_{-1}^{2, \alpha}\right)$. It is easy to see that the path $g_{\lambda}$ can be extended continuously to $[1, \infty]$, and since $v_{\lambda}$ tends to zero as $\lambda \rightarrow \infty$ for $r>r_{1}$ it follows that $g_{\infty} \in \mathcal{M}_{3}$.

Proposition 25. $\mathcal{M}_{3} / \mathcal{G}$ is connected to $\mathcal{M}_{4} / \mathcal{G}$.

Proof. Let $g \in \mathcal{M}_{3}$; choose $r_{0}>0$ so that $R, \beta$, and $v$ are supported in $B_{r_{0}}$, and let $\varphi(r)$ be a cut-off function as above. For $\lambda \in[1, \infty)$, define $\beta_{\lambda}=\varphi \phi_{1 / \lambda}^{*} \beta$ and $R_{\lambda}=\varphi \lambda^{-2} \phi_{1 / \lambda}^{*} R$ so that $R_{\lambda}, \beta_{\lambda}$ are always supported on $B_{r_{1}}$.

We now choose $v_{\lambda}$ to ensure $\bar{H}_{\lambda} \equiv \bar{H}\left(v_{\lambda}, \beta_{\lambda}\right)>0, \bar{\kappa}\left(v_{\lambda}\right)>0$. Define $\tilde{v}_{\lambda}=\phi_{1 / \lambda}^{*} v$, and let $\tilde{H}_{\lambda}=\bar{H}\left(\tilde{v}_{\lambda}, \tilde{\beta}_{\lambda}\right)$. By property (43) we have $\tilde{H}_{\lambda}=\phi_{1 / \lambda}^{*} \bar{H}$, and so $h \equiv \inf \bar{H}=\inf \tilde{H}_{\lambda}$ for all $\lambda \in[1, \infty)$. Let $r_{0}<r_{1}<r_{2}$, and let $0<\zeta(r)<1$ be supported on $B_{r_{2}}$ and satisfy $\zeta \equiv 1$ on $\left[0, r_{1}\right]$. Let $f(r)$ be a smooth non-negative function supported on $\left[r_{0}, r_{2}\right]$ satisfying on $\left[r_{0}, r_{1}\right]$ the inequality:

$$
f>-r^{-a-1}\left(\varphi h+r \varphi^{\prime}\right),
$$

where $a=\max \left\{-\left(2+2 r \partial_{r} \tilde{v}_{\lambda}\right), 0\right\}$. Let $\xi(r)=r^{a} \int_{r_{0}}^{r} f(s) d s, \psi=\xi+\varphi$. Define $v_{\lambda}=\zeta\left(\tilde{v}_{\lambda}+\frac{1}{2} \log \psi\right)$. Since $\xi \geq 0$, it now follows from (13) that we have for $r_{0}<r<r_{1}$ :

$$
\psi \bar{H}_{\lambda}=\xi\left(2+2 r \partial_{r} \tilde{v}_{\lambda}\right)+r \xi^{\prime}+\varphi \tilde{H}_{\lambda}+r \varphi^{\prime}>-a \xi+r \xi^{\prime}-r^{a+1} f=0 .
$$

Furthermore, since $\beta_{\lambda}=0$ in $B_{r_{2}} \backslash B_{r_{1}}$, we can also choose $\zeta$ so as to ensure that $\bar{H}_{\lambda}>0$ there, provided that $r_{2} / r_{1}$ is large enough. Finally, since $\psi, \zeta$ are radial, we have

$$
\begin{aligned}
e^{2 v_{\lambda}} \bar{\kappa}\left(v_{\lambda}\right) & =1-\not \boldsymbol{\phi}\left(\zeta \tilde{v}_{\lambda}+2^{-1} \zeta \log \psi\right)=1-\zeta \not \Delta \tilde{v}_{\lambda} \\
& =(1-\zeta)+\zeta e^{2 \tilde{v}_{\lambda}} \bar{\kappa}\left(\tilde{v}_{\lambda}\right)=(1-\zeta)+\zeta \phi_{1 / \lambda}^{*}\left(e^{2 v} \bar{\kappa}(g)\right)>0 .
\end{aligned}
$$


Define $g_{\lambda}=\lambda^{2} \phi_{1 / \lambda}^{*} g=g_{i j}(x / \lambda) d x^{i} d x^{j}$ in $B_{r_{0}}$ and note that with this definition the scaling remarks above show that $v_{\lambda}, \beta_{\lambda}, R_{\lambda}$ are smooth across $S_{r_{0}}$. As before, solve for $u_{\lambda}$ in $(4)$ on $\left[r_{0}, \infty\right) \times \mathbb{S}^{2}$ with initial data $\left.u_{\lambda}\right|_{S_{r_{0}}}$. Global existence, asymptotic behavior, and continuity are obtained from Theorems 14, 16, and 19 to give $g_{\lambda} \in C^{0}\left([1, \infty), C_{-1}^{2, \alpha}\right)$. Furthermore, since $\left.g_{i j}\right|_{0}=\delta_{i j}$, we have $\lim _{\lambda \rightarrow \infty}\left\|g_{i j}(x / \lambda)-\delta_{i j}\right\|_{C^{2}\left(B_{r_{2}}\right)}=0$ so that $\tilde{v}_{\lambda}, \beta_{\lambda}, R_{\lambda} \rightarrow$ 0 as $\lambda \rightarrow \infty$. Thus, as $\lambda \rightarrow \infty$ the family $g_{\lambda}$ converges, in $C_{-1}^{2, \alpha}$ norm, to the metric

$$
g_{\infty}=u_{\infty} d r^{2}+r^{2} e^{\zeta \log \psi} \bar{\gamma}_{A B} d \theta^{A} d \theta^{B},
$$

which satisfies $\left.u\right|_{B_{r_{0}}} \equiv 1, R\left(g_{\infty}\right) \equiv 0$. Since $g_{\infty}$ is both spherically symmetric and has vanishing scalar curvature we conclude that it is a flat metric.

However, note that $g_{1} \neq g$ since $v_{1}=\zeta\left(v+\frac{1}{2} \log \psi\right) \neq v$. In order to complete the proof of the lemma we now define a continuous path $g_{\lambda}$, $\lambda \in[0,1]$ in $\mathcal{M}_{3}$ between $g$ and $g_{1}$. In $B_{r_{0}}$ define $g_{\lambda}=g$. On $\mathbb{R}^{3} \backslash B_{r_{0}}$ define $\beta_{\lambda}=0, R_{\lambda}=0$ and $v_{\lambda}=(1-\lambda) v+\lambda v_{1}$. Then from Equations (13) and (14) and the fact that $\beta_{\lambda}=0$ on $\mathbb{R}^{3} \backslash B_{r_{0}}$ we have

$$
\begin{aligned}
\bar{H}_{\lambda} & =(1-\lambda) \bar{H}+\lambda \bar{H}_{1}>0 \\
e^{2 v_{\lambda}} \bar{\kappa}_{\lambda} & =(1-\lambda) e^{2 v} \bar{\kappa}+\lambda e^{2 v_{1}} \bar{\kappa}_{1}>0,
\end{aligned}
$$

and the quasi-convexity condition is preserved. It is also clear that $\operatorname{supp} v_{\lambda}$ is compact and thus $g_{\lambda}$ remains in $\mathcal{M}_{3}$.

\section{Appendix.}

In this appendix we prove the results stated in Section 3.1. All of the proofs in this appendix can be adapted to more general asymptotically flat manifolds with several ends, but for simplicity we present the simplest case: $M=\mathbb{R}^{3}$. Many of these results are quite standard, but since some are not readily available in the literature we prove them here for the sake of completeness. We thank Yanyan Li for several useful conversations on these topic. We begin with the elliptic estimate, Theorem 8 part (a), which is a straightforward corollary of the following proposition.

Proposition 26. Let $\tau>0$, and let $a^{i j}-\delta^{i j} \in C_{-\tau}^{k-1, \alpha}$ satisfy

$$
\Lambda^{-1}|\xi|^{2} \leq a^{i j} \xi_{i} \xi_{j} \leq \Lambda|\xi|^{2}
$$

for some $\Lambda>0$. Let $u \in C_{-\beta}^{0}$ be a classical solution of

$$
\partial_{i}\left(a^{i j} \partial_{j} u\right)=f
$$


with $f \in C_{-\beta-2}^{k-2, \alpha}$. Then

$$
\|u\|_{C_{-\beta}^{k, \alpha}} \leq C\left(\|f\|_{C_{-\beta-2}^{k-2, \alpha}}+\|u\|_{C_{-\beta}^{0}}\right) .
$$

Proof. From standard estimates on bounded domains, we can easily get that there is a constant $C$ such that:

$$
\|u\|_{C_{-\beta}^{k, \alpha}, B_{2}} \leq C\left(\|f\|_{C_{-\beta-2}^{k-2, \alpha}, B_{4}}+\|u\|_{C_{-\beta}^{0}, B_{4}}\right) ;
$$

see [10, Chapter 6]. Let $A=B_{5} \backslash B_{1}, A^{\prime}=B_{4} \backslash B_{2}$, and for each $n>1$ let $A_{n}=B_{5 \cdot 2^{n-1}} \backslash B_{2^{n-1}}, A_{n}^{\prime}=B_{2^{n+1}} \backslash B_{2^{n}}$. Then it follows easily that

$$
\begin{gathered}
\|w\|_{C_{-\beta}^{k, \alpha}, \mathbb{R}^{3} \backslash B_{2}} \leq 2 \sup _{n \geq 1}\|w\|_{C_{-\beta}^{k, \alpha}, A_{n}^{\prime}} \\
\|w\|_{C_{-\beta}^{k, \alpha}, \mathbb{R}^{3} \backslash B_{1}} \geq \sup _{n \geq 1}\|w\|_{C_{-\beta}^{k, \alpha}, A_{n}} .
\end{gathered}
$$

Furthermore, defining $w_{n}(x)=w\left(2^{n-1} x\right)$ we have

$$
\begin{aligned}
& C^{-1}\|w\|_{C_{-\beta}^{k, \alpha}, A_{n}} \leq 2^{(n-1) \beta}\left\|w_{n}\right\|_{C_{-\beta}^{k, \alpha}, A} \leq C\|w\|_{C_{-\beta}^{k, \alpha}, A_{n}}, \\
& C^{-1}\|w\|_{C_{-\beta}^{k, \alpha}, A_{n}^{\prime}} \leq 2^{(n-1) \beta}\left\|w_{n}\right\|_{C_{-\beta}^{k, \alpha}, A^{\prime}} \leq C\|w\|_{C_{-\beta}^{k, \alpha}, A_{n}^{\prime}} .
\end{aligned}
$$

Now the function $u_{n}$ satisfies on $A$ the equation:

$$
\partial_{i}\left(a_{n}^{i j} \partial_{j} u_{n}\right)=2^{2 n-2} f_{n},
$$

where the coefficients $a_{n}^{i j}$ satisfy

$$
\Lambda^{-1}|\xi|^{2} \leq a_{n}^{i j} \xi_{i} \xi_{j} \leq \Lambda|\xi|^{2}
$$

and

$$
\left\|a_{n}^{i j}\right\|_{C_{-\tau}^{k-1, \alpha}, A} \leq C\left(1+\left\|a^{i j}-\delta^{i j}\right\|_{C_{-\tau}^{k-1, \alpha}}\right) .
$$

Thus, as above, standard elliptic theory gives:

$$
\left\|u_{n}\right\|_{C_{-\beta}^{k, \alpha}, A^{\prime}} \leq C\left(\left\|2^{2 n-2} f_{n}\right\|_{C_{-\beta}^{k-2, \alpha}, A}+\left\|u_{n}\right\|_{C_{-\beta}^{0}, A}\right),
$$

where the constant $C$ depends only on $k, \alpha, \Lambda$, and $\left\|a^{i j}-\delta^{i j}\right\|_{C_{-\tau}^{k-1, \alpha}}$. In view of (50) and (51) this implies

$$
\|u\|_{C_{-\beta}^{k, \alpha}, A_{n}^{\prime}} \leq C\left(\|f\|_{C_{-\beta}^{k-2, \alpha}, A_{n}}+\|u\|_{C_{-\beta}^{0}, A_{n}}\right)
$$


Combining with (47), and using (48) and (49), we now obtain (46).

Proof of Theorem 8 part (a). Since

$$
\partial_{i}\left(\operatorname{det}(g) g^{i j} \partial_{j} u\right)=\operatorname{det}(g) \Delta_{g} u,
$$

Inequality (20) follows immediately from the proposition.

Unfortunately, the proof of part (b) of Theorem 8 given in [9] contains a gap. Indeed, the authors use the continuity method in their proof of surjectivity. However, they claim without proof that the set of operators $L=-\Delta_{g}+h$ which are injective is connected $^{2}$. Since this is false even for finite dimensional matrices, it seems to us unlikely to hold in a Banach space. Of course, this theorem is mostly applied when $h \geq 0$, in which case the connectedness statement above holds, and the proof given in [9] is valid. However, we prefer to present here a simple alternative proof of the original result as stated using Fredholm theory. The proof is based on the following compactness lemma from $[6]$ :

Lemma 27. The closed unit ball of $C_{-\beta}^{k, \alpha}$ is compact in $C_{-\beta^{\prime}}^{k, \alpha^{\prime}}$ provided $\alpha^{\prime}<$ $\alpha$ and $\beta^{\prime}<\beta$.

Proof. Let $u_{n}$ be a sequence in $C_{-\beta}^{k, \alpha}$ with $\left\|u_{n}\right\|_{C_{-\beta}^{k, \alpha}} \leq 1$. Since for each positive integer $N$ the space $C^{k, \alpha}\left(B_{N}\right)$ is compactly embedded in $C^{k, \alpha^{\prime}}\left(B_{N}\right)$, there is a subsequence, denoted again $u_{n}$, which converges in $C^{k, \alpha^{\prime}}\left(B_{N}\right)$ for every $N$. Let $u$ be the pointwise limit of $u_{n}$. Since $u_{n}$ converges to $u$, together with its derivatives up to order $k$, uniformly on each $B_{N}$, it follows immediately that $u \in C_{-\beta}^{k, \alpha}$ and $\|u\|_{C_{-\beta}^{k, \alpha}} \leq 1$. Now let $\epsilon>0$. Choose $N$ large enough so that $2 N^{\beta^{\prime}-\beta}<\epsilon$, and $n_{0}$ large enough that $\left\|u_{n}-u\right\|_{C_{-\beta^{\prime}}^{k, \alpha^{\prime}}, B_{N}}<\epsilon$ for all $n \geq n_{0}$. Then, we have:

$$
\begin{aligned}
\left\|u_{n}-u\right\|_{C_{-\beta^{\prime}}^{k, \alpha^{\prime}}} & =\max \left\{\left\|u_{n}-u\right\|_{C_{-\beta^{\prime}}^{k, \alpha^{\prime}}, B_{N}},\left\|u_{n}-u\right\|_{C_{-\beta^{\prime}}^{k, \alpha^{\prime}}, \mathbb{R}^{3} \backslash B_{N}}\right\} \\
& \leq \max \left\{\left\|u_{n}-u\right\|_{C_{-\beta^{\prime}}^{k, \alpha^{\prime}}, B_{N}}, N^{\beta^{\prime}-\beta}\left\|u_{n}-u\right\|_{C_{-\beta}^{k, \alpha^{\prime}}}\right\}<\epsilon .
\end{aligned}
$$

\footnotetext{
${ }^{2}$ In fact the authors consider more general operators, but the same considerations apply.
} 
Thus, $u_{n}$ converges to $u$ in $C_{\beta^{\prime}}^{k, \alpha^{\prime}}$.

We will also use the fact that $\Delta_{g}: C_{-\beta}^{k, \alpha} \rightarrow C_{-\beta-2}^{k-2, \alpha}$ is an isomorphism.

Proposition 28. Let $\tau>0,0<\beta<1$, and let $g \in \delta+C_{-\tau}^{k-1, \alpha}$ be a metric on $\mathbb{R}^{3}$. Then the operator $\Delta_{g}: C_{-\beta}^{k, \alpha} \rightarrow C_{-\beta-2}^{k-2, \alpha}$ is an isomorphism.

Proof. The injectivity of $\Delta_{g}$ follows from the maximum principle. The solvability of the equation $\Delta_{g} u=f$ is standard; see for example [16]. We now estimate $u$ in $C_{-\beta}^{0}$ using the representation (21) and the estimate (22) on $G$ :

$$
|u(x)| \leq C\|f\|_{C_{-2-\beta}^{0}} \int_{\mathbb{R}^{3}} \frac{d y}{|y|^{2+\beta}|x-y|} .
$$

We break up the integral on the right-hand side into three parts and estimate each part by $C|x|^{-\beta}$. First on $\Omega_{1}=\{|x-y| \geq 2|x|\}$ we have $|y| \geq|x-y| / 2$; hence

$$
\int_{\Omega_{1}} \frac{d y}{|y|^{2+\beta}|x-y|} \leq C \int_{\Omega_{1}} \frac{d y}{|x-y|^{3+\beta}}=C \int_{\mathbb{R}^{3} \backslash B_{2|x|}} \frac{d y}{|y|^{3+\beta}}=\frac{C}{|x|^{\beta}} .
$$

Next, on $\Omega_{2}=\{|x| / 2 \leq|x-y| \leq 2|x|\}$, we have $|y| \leq 3|x-y|$, and furthermore $\Omega_{2} \subset B_{3|x|}$; hence

$$
\int_{\Omega_{2}} \frac{d y}{|y|^{2+\beta}|x-y|} \leq C \int_{\Omega_{2}} \frac{d y}{|y|^{3+\beta}} \leq C \int_{B_{3|x|}} \frac{d y}{|y|^{3+\beta}}=\frac{C}{|x|^{\beta}} .
$$

Finally, on $\Omega_{3}=\{|x-y| \leq|x| / 2\}$, we have $|y| \geq|x| / 2$; hence

$$
\int_{\Omega_{3}} \frac{d y}{|y|^{2+\beta}|x-y|} \leq \frac{C}{|x|^{2+\beta}} \int_{\Omega_{3}} \frac{d y}{|x-y|}=\frac{C}{|x|^{2+\beta}} \int_{B_{|x| / 2}} \frac{d y}{|y|}=\frac{C}{|x|^{\beta}}
$$

This shows that

$$
|x|^{\beta}|u(x)| \leq C\|f\|_{C_{-\beta}^{0}} .
$$

Applying this inequality after having performed a translation on $\mathbb{R}^{3}$ by a vector of length 2 , we also get a supremum bound for $u$ on $B_{1}$, and hence a bound on $\|u\|_{C_{-\beta}^{0}}$. Once this bound for $u$ is established, the estimate (20) shows that $u \in C_{-\beta}^{k, \alpha}$, and thus $\Delta_{g}$ is surjective. The boundedness of the inverse follows from the compactness lemma. Indeed, suppose the inverse is not bounded. 
Then there is a sequence $u_{n}$ with $\left\|u_{n}\right\|_{C_{-\beta}^{k, \alpha}}=1$ and $\left\|\Delta_{g} u_{n}\right\|_{C_{-\beta-2}^{k-\alpha}} \rightarrow 0$. By Lemma 27, we may assume that $\left\|u_{n}-u\right\|_{C_{-\beta^{\prime}}^{k, \alpha^{\prime}}} \rightarrow 0$ for some function $u \in C_{-\beta}^{k, \alpha}$ and $\alpha^{\prime}<\alpha, \beta^{\prime}<\beta$. Clearly, $\Delta_{g} u=0$ in contradiction to the injectivity of $\Delta_{g}$.

Proof of Theorem 8 part (b). Let $K: C_{-\beta}^{k, \alpha} \rightarrow C_{-\beta}^{k, \alpha}$ be defined by $K u=$ $\Delta_{g}^{-1} h u$. We claim that $K$ is compact. Indeed, if we take $0<\epsilon<\nu-2$, the inclusion $\iota: C_{-\beta}^{k, \alpha} \rightarrow C_{-\beta+\epsilon}^{k-2, \alpha}$ is compact by the lemma, and the multiplication operator $h: C_{-\beta+\epsilon}^{k-2, \alpha} \rightarrow C_{-\beta-2}^{k-2, \alpha}$ is bounded; thus $K=\Delta_{g}^{-1} \cdot h \cdot \iota$ is compact. It follows that $I+K$ is Fredholm of index zero [11, Theorem 3.17]. Since $I+K=\Delta_{g}^{-1} L$, the injectivity of $L$ implies the injectivity of $I+K$; whence $I+K$ is also surjective. Now, a simple argument as in the previous proof shows that $I+K$ has a bounded inverse. Indeed, if it didn't then there would be a sequence $u_{n}$ with $\left\|u_{n}\right\|=1$ and $\left\|(I+K) u_{n}\right\| \rightarrow 0$. Since $K$ is compact, we could assume that $K u_{n} \rightarrow v$, which implies that $u_{n} \rightarrow-v$, and thus $(I+K) v=0$, in contradiction to $\operatorname{ker}(I+K)=0$. This shows that $I+K$ is an isomorphism. Therefore, we conclude that $L=\Delta_{g}(I+K)$ is also an isomorphism.

Before giving the proof of Theorem 9, we first establish the corresponding elliptic estimate.

Proposition 29. Let $\beta, \tau>0$, and let $g \in \delta+C_{-\tau}^{k-1, \alpha}$ be a metric on $\mathbb{R}^{3}$. If $u \in C_{-\beta}^{0}\left(\mathbb{R}^{3}\right)$ and $\Delta_{g} u \in D_{-3}^{k-2, \alpha}$ then $u \in E_{-1}^{k, \alpha}$ and

$$
\|u\|_{C_{-1}^{k, \alpha}} \leq C\left\|\Delta_{g} u\right\|_{D_{-3}^{k-2, \alpha}}
$$

Furthermore, $\Delta_{g}: E_{-1}^{k, \alpha} \rightarrow D_{-3}^{k-2, \alpha}$ is an isomorphism.

Proof. We first establish a bound for $u$ in $C_{-1}^{0}$ in terms of $f=\Delta_{g} u$ in $D_{-3}^{0, \alpha}$. This bound is obtained from the representation (21) and the estimate (22) on $G$, as in the proof of Proposition 28:

$$
|u(x)| \leq C \int_{\mathbb{R}^{3}} \frac{|f(y)| d y}{|x-y|} .
$$


Here we break up the integral on the right-hand side into two parts. On $\Omega_{1}=\{|x-y| \geq|x| / 2\}$, we have

$$
\int_{\Omega_{1}} \frac{|f(x)| d y}{|x-y|} \leq \frac{2}{|x|}\|f\|_{L^{1}}
$$

On $\Omega_{2}=\{|x-y| \leq|x| / 2\}$, we have $|y| \geq|x| / 2$; hence

$$
\int_{\Omega_{2}} \frac{|f(x)| d y}{|x-y|} \leq\|f\|_{C_{-3}^{0}} \int_{\Omega_{2}} \frac{d y}{|y|^{3}|x-y|} \leq \frac{8\|f\|_{C_{-3}^{0}}}{|x|^{3}} \int_{B_{|x| / 2}} \frac{d y}{|y|}=\frac{C\|f\|_{C_{-3}^{0}}}{|x|} .
$$

This shows that

$$
|x||u(x)| \leq C\|f\|_{D_{-3}^{0, \alpha}} .
$$

Applying this inequality after having performed a translation on $\mathbb{R}^{3}$ by a vector of length 2 , we also get a supremum bound for $u$ on $B_{1}$, and hence a bound on $\|u\|_{C_{-1}^{0}}$. Now, (53) follows from Theorem 8 part (a), Inequality (20). We have $\Delta_{g} u \in L^{1}$ and

$$
\Delta_{g} u-\Delta u=\left(g^{i j}-\delta^{i j}\right) \partial_{i} \partial_{j} u-g^{i j} \Gamma_{i j}^{k} \partial_{k} u,
$$

where $\Delta=\Delta_{\delta}$. Thus, we obtain

$$
\left\|\Delta_{g} u-\Delta u\right\|_{L^{1}} \leq C\|u\|_{C_{-1}^{2}}
$$

and it follows that $\Delta u \in L^{1}$ and $u \in E_{-1}^{k, \alpha}$. Inequality (53) implies that $\Delta_{g}$ is injective with a bounded inverse, and (21) implies surjectivity.

Finally, we can now prove the isomorphism theorem in $E_{-1}^{k, \alpha}$. The proof is similar to the proof of part (b) of Theorem 8, with minor changes necessary to keep track of the condition $\Delta u \in L^{1}$.

Proof of Theorem 9. First, note that if $u \in E_{-1}^{k, \alpha}$ then $\Delta_{g} u \in L^{1}$ in view of (55). Furthermore, $h u \in L^{1}$ since

$$
\|h u\|_{L^{1}} \leq\left\|\sigma^{-\nu-1}\right\|_{L^{1}}\|h\|_{C_{-\nu}^{0}}\|u\|_{C_{-1}^{0}}
$$

Thus, $\left(\Delta_{g}-h\right) u \in L^{1}$; hence $\Delta_{g}-h$ indeed maps $E_{-1}^{k, \alpha}$ continuously into $D_{-3}^{k-2, \alpha}$. The proof of part (b) of Theorem 8 can now be applied once the operator $K: u \mapsto \Delta_{g}^{-1} h u$ is shown to map $E_{-1}^{k, \alpha}$ into itself compactly. Equivalently, it is enough to verify that the multiplication operator 
$h: E_{-1}^{k, \alpha} \rightarrow D_{-3}^{k-2, \alpha}$ is compact. Let $u_{n}$ be a bounded sequence in $E_{-1}^{k, \alpha}$. From the proof of Theorem 8 we know that there is a subsequence, denoted again by $u_{n}$, such that $h u_{n}$ converges in $C_{-3}^{k-2, \alpha}$. It remains to show that $h u_{n}$ contains a subsequence which converges in $L^{1}$. This follows from a minor refinement of (56). Indeed, if $3-\nu<\beta<1$ then by Lemma $27 u_{n}$ contains a subsequence that converges in $C_{-\beta}^{0}$ and since

$$
\|h u\|_{L^{1}} \leq\left\|\sigma^{-\nu-\beta}\right\|_{L^{1}}\|h\|_{C_{-\nu}^{0}}\|u\|_{C_{-\beta}^{0}}
$$

we have that this subsequence converges in $L^{1}$.

Finally, we give the proof of Lemma 10.

Proof of Lemma 10. Without loss of generality we assume that $y=0$, and we let $u(x)=G(x, 0)$. Define $\left(g_{n}\right)_{i j}(x)=g_{i j}\left(2^{n-1} x\right)$ and $u_{n}(x)=u\left(2^{n-1} x\right)$ as in the proof of Proposition 26, but now for any integer $n$. Then $\Delta_{g_{n}} u_{n}=0$ on $\mathbb{R}^{3} \backslash 0$. Standard elliptic theory [10, Chapter 6$]$ combined with the supremum bound (22) on $G$ gives:

$$
\left\|u_{n}\right\|_{C^{2}, A^{\prime}} \leq C\left\|u_{n}\right\|_{C^{0}, A} \leq C 2^{1-n},
$$

where $A=B_{5} \backslash B_{1}, A^{\prime}=B_{4} \backslash B_{2}$, and the constant $C$ is independent of $n$. It follows that we have on $A_{n}^{\prime}=B_{2^{n+1}} \backslash B_{2^{n}}$ :

$$
\|u(x)\|_{C^{0}, A_{n}^{\prime}}+\|r \partial u(x)\|_{C^{0}, A_{n}^{\prime}}+\left\|r^{2} \partial^{2} u(x)\right\|_{C^{0}, A_{n}^{\prime}} \leq C 2^{1-n} \leq \frac{C}{|x|},
$$

where $r=|x|$. This implies

$$
\begin{aligned}
|\partial G(x, 0)| & \leq \frac{C}{|x|^{2}} \\
\left|\partial^{2} G(x, 0)\right| & \leq \frac{C}{|x|^{3}},
\end{aligned}
$$

which completes the proof.

\section{References.}

[1] R. BARTNIK, Existence of maximal surfaces in asymptotically flat spacetimes, Comm. Math. Phys. 94 No. 2. (1984) 155-175.

[2] R. BARTNIK, Quasi-spherical metrics and prescribed scalar curvature, J. Differential Geom. 37 No. 1 (1993) 31-71. 
[3] H. BRAy, Proof of the Riemannian Penrose conjecture using the positive mass theorem, J. Differential Geom. 59 (2001) 177-267.

[4] M. Cantor, A necessary and sufficient condition for York data to specify an asymptotically flat spacetime, J. Math. Phys. 20 (1979) 17411744.

[5] M. CAntor AND D. BRILL, The laplacian on asymptotically flat manifolds and the specification of scalar curvature, Comp. Math. 43 No. 3 (1981) 317-330.

[6] A. Chaluub-Simon and Y. Choquet-Bruhat, Problèmes elliptiques du second ordre sur une variété euclidienne à l'infini, Ann. Fac. Sci. Toulouse Math. (5) 1 (1979), No. 1, 9-25.

[7] D. Christodoulou and S. Klainerman, The Global Nonlinear Stabililty of Minkowski Space, No. 41, Princeton University Press, Princeton, 1993.

[8] D. Cristodoulou and N. O'Murchadha, The boost problem in genreral relativity, Comm. in Math. Phys. 80 No. 2. (1981) 271-300.

[9] Y. Choquet-Bruhat, Global solutions of the constraints equations on open and closed manifolds, Gen. Relativity Gravitation 5 No. 1 (1974) 49-60.

[10] D. Gilbarg; N. S. Truginger, Elliptic Partial Differential Equations of Second Order Second Edition, Spriger-Verlag, New York 1983.

[11] D. E. Edmunds And W. D. Evans, Spectral Theory of Differential Operators, Oxford Mathematical Monographs, Oxford Science Publications, Clarendon Press, Oxford, 1987.

[12] A. Friedman, Partial Differential Equations of Parabolic Type Prentice-Hall, Inc., Englewood Cliffs, N. J.

[13] O. A. Ladyženskaja, V.A Solonnikov and N. N. Ural'Ceva, Linear and Qausilinear Equations of Parabolic Type, Trans. of Math. Mon., Vol. 23, AMS, Rhode Island, 1968.

[14] J. M. Lee And T. H. Parker, The Yamabe Problem, Bull. of the AMS 17 No. 1 (1987) 37-92.

[15] G. Lieberman, Second Order Parabolic Partial Differential Equations, World Scientiic, New Jersey, 1996 
[16] J. SERrin And H. F. Weinberger, Isolated singularities of solutions of linear elliptic equations, Amer. J. Math. 88 (1966) 258-272.

[17] B. Smith, Asymptotically flat quasi-convex Riemannian metrics of nonnegative scalar curvature and the constraint equations in general relativity, Ph.D. Thesis, University of Alabama at Birmingham, 2001.

[18] B. Smith And G. Weinstein, On the connectedness of the space of initial data for the Einstein equations, Electron. Res. Announc. Amer. Math. Soc. 6 (2000) 52-63.

[19] J. Smoller, Shock Waves and Reaction Diffusion Equations Second Edition, Springer-Verlag, New York 1994.

[20] Robert M. Wald, General Relativity, The University of Chicago Press, Chicago 1984

[21] J. W. York JR., Gravitational degrees of freedom and the initial-value problem, Phys. Rev. Lett. 23 No. 26 (1971) 1656-1658.

[22] J. W. YorK JR., Role of conformal three-geometry in the dynamics of gravitation, 28 (1971) 1082-1085.

Received May 2002. 